\title{
A fast second-order implicit difference method for time-space fractional advection-diffusion equation
}

\author{
Yong-Liang Zhao ${ }^{a}$, Ting-Zhu Huang ${ }^{a} \dagger$ Xian-Ming $\mathrm{Gu}^{b} \ddagger$ Wei-Hua Luo ${ }^{c} \S$ \\ a. School of Mathematical Sciences, \\ University of Electronic Science and Technology of China, \\ Chengdu, Sichuan 611731, P.R. China \\ b. School of Economic Mathematics, \\ Southwestern University of Finance and Economics, \\ Chengdu, Sichuan 611130, P.R. China \\ c. Data Recovery Key Laboratory of Sichuan Province, \\ Neijiang Normal University, Neijiang, Sichuan, 641100, P.R. China
}

\begin{abstract}
In this paper, we consider a fast and second-order implicit difference method to approximate a class of linear time-space fractional variable coefficients advection-diffusion equation. To begin with, we construct an implicit difference scheme based on $L 2-1_{\sigma}$ formula [Alikhanov AA. 2015;280:424-38.] for the temporal discretization and weighted and shifted Grünwald method for the spatial discretization. Then, unconditional stability of the implicit difference scheme is proved, and we theoretically and numerically show that it converges in the $L_{2}$-norm with the optimal order $\mathcal{O}\left(\tau^{2}+h^{2}\right)$ with time step $\tau$ and mesh size $h$. Moreover, we utilize the same measure to solve the nonlinear case of this problem. For purpose of effectively solving the discretized systems with the Toeplitz matrix, two fast Krylov subspace solvers with suitable circulant preconditioners are designed. In each iterative step, these methods reduce the storage requirements of the resulting equations from $\mathcal{O}\left(N^{2}\right)$ to $\mathcal{O}(N)$ and the computational complexity from $\mathcal{O}\left(N^{3}\right)$ to $\mathcal{O}(N \log N)$, where $N$ is the number of grid nodes. Numerical experiments are carried out to demonstrate that these methods are more practical than the traditional direct solvers of the implicit difference methods, in terms of memory requirement and calculation time.

Key words: Linear/Nonlinear fractional advection-diffusion equation; Weighted and shifted Grünwald scheme; $L 2-1_{\sigma}$ formula; Krylov subspace method; Toeplitz matrix; Fast Fourier transform; Circulant preconditioner.
\end{abstract}

\section{Introduction}

\footnotetext{
*E-mail address: uestc_ylzhao@sina.com

${ }^{\dagger}$ Corresponding author. E-mail address: tingzhuhuang@126.com. Tel: +86 2861831608, fax: +86 28 61831280.

${ }^{\ddagger}$ E-mail address: guxianming@live.cn, x.m.gu@rug.nl

$\S$ E-mail address: huaweiluo2012@163.com
} 
This manuscropt is concerned with a fast second-order implicit difference method (IDM) for solving the initial-boundary value problem of the time-space fractional advection-diffusion equation (TSFADE) [1,2]:

$$
\left\{\begin{aligned}
D_{0, t}^{\theta} u(x, t)= & -d_{+}(t) D_{0, x}^{\alpha} u(x, t)-d_{-}(t) D_{x, L}^{\alpha} u(x, t) \\
& +e_{+}(t) D_{0, x}^{\beta} u(x, t)+e_{-}(t) D_{x, L}^{\beta} u(x, t)+f(x, t), \\
u(x, 0)=u_{0}(x), & 0 \leq x \leq L, \\
u(0, t)=u(L, t)=0, & 0 \leq t \leq T,
\end{aligned}\right.
$$

where $\theta, \alpha \in(0,1], \beta \in(1,2], 0<x<L$, and $0<t \leq T$. Here, the parameters $\theta, \alpha$ and $\beta$ are the order of the TSFADE, $f(x, t)$ is the source term, and diffusion coefficient functions $d_{ \pm}(t)$ and $e_{ \pm}(t)$ are non-negative under the assumption that the flow is from left to right. The TSFADE (1.1) can be regarded as generalizations of classical advection-diffusion equations with the first-order time derivative replaced by the Caputo fractional derivative of order $\theta \in(0,1]$, and the first-order and second-order space derivatives replaced by the two-sided Riemann-Liouville fractional derivatives of order $\alpha \in(0,1]$ and $\beta \in(1,2]$. Namely, the time fractional derivative in (1.1) is the Caputo fractional derivative [3, 4] of order $\theta$ denoted by

$$
D_{0, t}^{\theta} u(x, t)=\frac{1}{\Gamma(1-\theta)} \int_{0}^{t} \frac{\partial u(x, \xi)}{\partial \xi} \frac{d \xi}{(t-\xi)^{\theta}},
$$

and the left $\left(D_{0, x}^{\alpha}, D_{0, x}^{\beta}\right)$ and right $\left(D_{x, L}^{\alpha}, D_{x, L}^{\beta}\right)$ space fractional derivatives in (1.1) are the Riemann-Liouville fractional derivatives [3, 4] defined as

(1) left Riemann-Liouville fractional derivative:

$$
D_{0, x}^{\gamma} u(x, t)=\frac{1}{\Gamma(n-\gamma)} \frac{d^{n}}{d x^{n}} \int_{0}^{x} \frac{u(\eta, t)}{(x-\eta)^{\gamma-n+1}} d \eta,
$$

(2) right Riemann-Liouville fractional derivative:

$$
D_{x, L}^{\gamma} u(x, t)=\frac{(-1)^{n}}{\Gamma(n-\gamma)} \frac{d^{n}}{d x^{n}} \int_{x}^{L} \frac{u(\eta, t)}{(\eta-x)^{\gamma-n+1}} d \eta,
$$

where $\Gamma(\cdot)$ denotes the Gamma function and $n-1 \leq \gamma<n$ ( $n$ is a positive integer). In reality, when $\theta=\alpha=1$ and $\beta=2$, the above equation (1.1) reduces to the classical advection-diffusion equation (ADE).

There have been many studies and applications of the fractional ADE. It should be point out that the fractional ADE provides more adequate and accurate description of the movement of solute in an aquifer than traditional second-order ADEs do [5, 6], and also used to approach the description of transport dynamics in complex systems governed by anomalous diffusion and non-exponential relaxation patterns [7. Moreover, the fractional ADE is a more suitable model for many problems, such as engineering, chemistry, entropy [8] and hydrology [9]. To obtain the analytical solutions of fractional partial differential equations [3], numerous analytical methods, such as the Fourier transform method, Adomians decomposition method, the Laplace transform method, shifted Legendre polynomials [10] and the Mellin transform method, have been developed. Since there are very few cases in which the closed-form analytical solutions are available, or the obtained analytical solutions are less practical (expressed by the transcendental 
functions or infinite series). Researches on numerical approximation and techniques for the solution of fractional difference equations (FDEs) have attracted intensive interest; see [11 19] and references therein.

Regarding numerical methods for fractional advection-diffusion problems. In the last years, most early established numerical methods are developed for handling the space or time fractional ADE. For the time fractional ADE, many early implicit numerical methods are derived by the combination of the $L 1$ approximate formula [20,21]. These methods are unconditionally stable, but the time accuracy can not meet second order. In addition, some other related numerical methods have already been proposed for handling the time fractional ADE; see [25] 27] for details. On the other hand, for the space fractional ADE, many researchers exploited the conventional shifted Grünwald discretization [22] and the implicit Euler (or Crank-Nicolson) time-step discretization for two-sided Riemann-Liouville fractional derivatives and the first order time derivative, respectively. Later, Sousa and Li [23] derived a weighted finite difference scheme for producing the novel numerical methods, which achieved the second order accuracy in both time and space for space fractional ADE. Qu and Lei et al. employed circulant and skewcirculant splitting iteration, which could reduce the required algorithm storage, for implementing the above mentioned second order numerical method; see [24] for details.

Contrarily, although the numerical methods for space or time fractional ADE were extensively investigated in the past researches, the work about numerically handling the TSFADE is not too much. Firstly, Liu et al. [22] and Zhang [28,29] worked out a series of studies about constructing the implicit difference scheme (IDS) for TSFADE, however all these numerical schemes achieve the convergence with first order accuracy in both space and time from both the theoretical and numerical perspectives. Liu et al. [1] also considered a space-time fractional advection dispersion equation and the implicit proposed difference method has convergence $\mathcal{O}(\tau+h)$. Then, Shen et al. 30] presented an explicit difference approximation with convergence $\mathcal{O}(\tau+h)$ and an implicit difference approximation with convergence rate $\mathcal{O}\left(\tau^{2-\alpha}+h\right)$. Inspired by Liu's paper [1, Zhao et al. 2] introduced preconditioned iterative methods to reduce the amount of computation while solving TSFADE, but its convergence can only reach $\mathcal{O}\left(\tau^{2-\alpha}+h\right)$. Later, $\mathrm{Gu}$ et al. [50] established fast solution techniques to solve the discretization linear system of the time-space fractional convection-diffusion equation, which has convergence rate $\mathcal{O}\left(\tau^{2}+h^{2}\right)$. Furthermore, most of these methods have no complete theoretical analysis for both stability and convergence, refer to [31 34] for details.

Since the fractional differential operator is nonlocal, it shows that a naive discretization of the FDE, even though implicit, leads to unconditionally unstable [14, 15]. Moreover, traditional methods for solving FDEs tend to generate full coefficient matrices, which require computational cost of $\mathcal{O}\left(N^{3}\right)$ and storage of $\mathcal{O}\left(N^{2}\right)$ [35. There is no doubt that if $N$ is not small, both calculation time and storage requirements are tremendous, and this situation is that we try to avoid. To optimize the computational complexity, Meerschaet and Tadjeran [14,15] proposed a shifted Grünwald discretization to approximate FDEs, which has been proved to be unconditionally stable. Later, Wang, Wang and Sircar [36] discovered that the full coefficient matrix derived by the shifted Grünwald scheme holds a Toeplitz-like structure. More precisely, this matrix can be written as a sum of diagonal-multiply-Toeplitz matrices. This means that the matrixvector multiplication for Toeplitz matrix can be computed by the fast Fourier transform (FFT) with $\mathcal{O}(N \log N)$ operations [37, and the storage requirement reduces to $\mathcal{O}(N)$. Thanks to the Toeplitz-like structure, Wang and Wang [38] employed the CGNR method to solve discretized linear system of FDE by the Meerschaet-Tadjeran's method, and the cost of each iteration is 
$\mathcal{O}(N \log N)$. Numerical experiments indicate that the CGNR method is fast when the diffusion coefficients are small enough, namely, the discretized system is well-conditioned. Nevertheless, the discretized system will become ill-conditioned when the diffusion coefficients are not small, hence the CGNR method converges very slowly. To overcome this shortcoming, Zhao et al. extended the precondition technique, for handling the Toeplitz-like matrix with structure as the sum of diagonal-multiply-Toeplitz matrices [2. Their results are related to the promising acceleration of the convergence of the iterative methods, while solving (1.1), see [39, 40] for other preconditioning methods. For the same reason, we propose two preconditioned iterative methods, i.e., the preconditioned biconjugate gradient stabilized (BiCGSTAB) method [41] and the preconditioned generalized product-type solvers based on $\operatorname{BiCOR}(\operatorname{GPBiCOR}(m, \ell))$ method [42, and observe results related to the acceleration of the convergence of the iterative methods to solve (1.1).

The rest of this paper is arranged as follows. In Section 2, the implicit difference scheme for (1.1) is presented, and we prove that this scheme is unconditionally stable and convergent with

the accuracy of $\mathcal{O}\left(\tau^{2}+h^{2}\right)$. Then, we extend the problem to nonlinear situation through utilizing the same technique. In Section 3, the $\operatorname{BiCGSTAB}$ method and the $\operatorname{GPBiCOR}(m, \ell)$ method with suitable circulant preconditioners are proposed to solve the discretized system. In Section 4, numerical results are reported to demonstrate the efficiency of our numerical approaches, and concluding remarks are given in Section 5.

\section{An implicit difference scheme for TSFADE}

In this section, we present an implicit difference method for discretizing the TSFADE defined by (1.1). Unlike the former numerical approaches [1, 2, 30, we exploit henceforth two-sided fractional derivatives to approximate the Riemann-Liouville derivatives in (1.3) and (1.4). we can show that, by two-sided fractional derivatives, this proposed method is also unconditionally stable and convergent second order accuracy in both time and space.

\subsection{Discretization of the TSFADE}

In order to derive the proposed scheme, we first introduce the mesh $\bar{\omega}_{h \tau}=\bar{\omega}_{h} \times \bar{\omega}_{\tau}$, where $\bar{\omega}_{h}=\left\{x_{i}=i h, i=0,1, \cdots, N ; x_{0}=0, x_{N}=L\right\}$ and $\bar{\omega}_{\tau}=\left\{t_{j}=j \tau, j=0,1, \cdots, M ; t_{M}=T\right\}$. Besides, $\boldsymbol{v}=\left\{v_{i} \mid 0 \leq i \leq N\right\}$ be any grid function. Then the following lemma introduced in [43] gives a description on the Caputo fractional derivative discretization.

Lemma 2.1. suppose $0<\theta<1, \sigma=1-\frac{\theta}{2}, y(t) \in \mathcal{C}^{3}[0, T]$, and $t_{j+\sigma}=(j+\sigma) \tau$. Then

$$
\left|D_{0, t}^{\theta} y(t)-\Delta_{0, t_{j+\sigma}}^{\theta} y(t)\right|=\mathcal{O}\left(\tau^{3-\theta}\right),
$$

where

$$
\Delta_{0, t_{j+\sigma}}^{\theta} y(t)=\frac{\tau^{-\theta}}{\Gamma(2-\theta)} \sum_{s=0}^{j} c_{j-s}^{(\theta, \sigma)}\left[y\left(t_{s+1}\right)-y\left(t_{s}\right)\right],
$$


and for $j=0, c_{0}^{(\theta, \sigma)}=a_{0}^{(\theta, \sigma)}$, for $j \geq 1$,

$$
c_{s}^{(\theta, \sigma)}= \begin{cases}a_{0}^{(\theta, \sigma)}+b_{1}^{(\theta, \sigma)}, & s=0, \\ a_{s}^{(\theta, \sigma)}+b_{s+1}^{(\theta, \sigma)}-b_{s}^{(\theta, \sigma)}, & 1 \leq s \leq j-1, \\ a_{j}^{(\theta, \sigma)}-b_{j}^{(\theta, \sigma)}, & s=j,\end{cases}
$$

in which $a_{0}^{(\theta, \sigma)}=\sigma^{1-\theta}, a_{l}^{(\theta, \sigma)}=(l+\sigma)^{1-\theta}-(l-1+\sigma)^{1-\theta}$, for $l \geq 1$; and $b_{l}^{(\theta, \sigma)}=\frac{1}{2-\theta}[(l+$ $\left.\sigma)^{2-\theta}-(l-1+\sigma)^{2-\theta}\right]-\frac{1}{2}\left[(l+\sigma)^{1-\theta}-(l-1+\sigma)^{1-\theta}\right]$.

On the other hand, the Riemann-Liouville derivatives (1.3) and (1.4) can be approximated by the weighted and shifted Grünwald difference (WSGD) operator in Deng's paper [44] (in this paper $(p, q)=(1,0))$, for parameter $\gamma$, i.e.,

$$
\begin{gathered}
D_{0, x}^{\gamma} u(x, t)=\frac{1}{h^{\gamma}} \sum_{k=0}^{\left[\frac{x}{h}\right]+1} \omega_{k}^{(\gamma)} u(x-(k-1) h, t)+\mathcal{O}\left(h^{2}\right), \\
D_{x, L}^{\gamma} u(x, t)=\frac{1}{h^{\gamma}} \sum_{k=0}^{\left[\frac{L-x}{h}\right]+1} \omega_{k}^{(\gamma)} u(x+(k-1) h, t)+\mathcal{O}\left(h^{2}\right) .
\end{gathered}
$$

Let $u_{i}^{j}$ represent the numerical approximation of $u\left(x_{i}, t_{j}\right)$, then we denote that

$$
\delta_{x,+}^{\gamma} u_{i}^{j}=\frac{1}{h^{\gamma}} \sum_{k=0}^{i+1} \omega_{k}^{(\gamma)} u_{i-k+1}^{j}, \quad \delta_{x,-}^{\gamma} u_{i}^{j}=\frac{1}{h^{\gamma}} \sum_{k=0}^{N-i+1} \omega_{k}^{(\gamma)} u_{i+k-1}^{j},
$$

where

$$
\omega_{0}^{(\gamma)}=\frac{\gamma}{2} g_{0}^{(\gamma)}, \quad \omega_{k}^{(\gamma)}=\frac{\gamma}{2} g_{k}^{(\gamma)}+\frac{2-\gamma}{2} g_{k-1}^{(\gamma)}, k \geq 1
$$

and

$$
g_{0}^{(\gamma)}=1, \quad g_{k}^{(\gamma)}=\left(1-\frac{\gamma+1}{k}\right) g_{k-1}^{(\gamma)}, k=1,2, \ldots
$$

Suppose $u(x, t) \in \mathcal{C}_{x, t}^{4,3}[0, L] \times[0, T]$ is a solution of the TSADE (1.1). For simplicity, we introduce some symbols

$$
\begin{gathered}
u_{i}^{(j+\sigma)}=\sigma u_{i}^{j+1}+(1-\sigma) u_{i}^{j}, \quad d_{ \pm}^{j+\sigma}=d_{ \pm}\left(t_{j+\sigma}\right), \quad e_{ \pm}^{j+\sigma}=e_{ \pm}\left(t_{j+\sigma}\right), \quad f_{i}^{j+\sigma}=f\left(x_{i}, t_{j+\sigma}\right), \\
\delta_{h}^{\alpha, \beta} u_{i}^{(j+\sigma)}=-d_{+}^{j+\sigma} \delta_{x,+}^{\alpha} u_{i}^{(j+\sigma)}-d_{-}^{j+\sigma} \delta_{x,-}^{\alpha} u_{i}^{(j+\sigma)}+e_{+}^{j+\sigma} \delta_{x,+}^{\beta} u_{i}^{(j+\sigma)}+e_{-}^{j+\sigma} \delta_{x,-}^{\beta} u_{i}^{(j+\sigma)} .
\end{gathered}
$$

Using (2.1)-(2.3), we shall see that the solution of (1.1) can be approximated by the following implicit difference scheme for $(x, t)=\left(x_{i}, t_{j+\sigma}\right) \in \bar{\omega}_{h \tau}, i=1,2, \ldots, N-1, j=0,1, \ldots, M-1$ :

$$
\Delta_{0, t_{j+\sigma}}^{\theta} u_{i}=\delta_{h}^{\alpha, \beta} u_{i}^{(j+\sigma)}+f_{i}^{j+\sigma} .
$$

Then we derive the implicit difference scheme with the approximation order $\mathcal{O}\left(\tau^{2}+h^{2}\right)$ :

$$
\begin{cases}\Delta_{0, t_{j+\sigma}}^{\theta} u_{i}=\delta_{h}^{\alpha, \beta} u_{i}^{(j+\sigma)}+f_{i}^{j+\sigma}, & 1 \leq i \leq N-1, \quad 0 \leq j \leq M-1, \\ u_{i}^{0}=u_{0}\left(x_{i}\right), & 1 \leq i \leq N-1, \\ u_{0}^{j}=u_{N}^{j}=0, & 0 \leq j \leq M-1 .\end{cases}
$$

It is interesting to note that for $\theta \rightarrow 1$, we obtain the Crank-Nicolson difference scheme. 


\subsection{Analysis of the implicit difference scheme}

In this subsection, we analyze the stability and convergence for the implicit difference scheme (2.4). Before proving the stability and convergence of the implicit difference scheme (2.4), we provide some properties of the coefficients $\omega_{k}^{(\alpha)}$ and $\omega_{k}^{(\beta)}$, which are derived from $g_{k}^{(\alpha)}$ and $g_{k}^{(\beta)}[1,2,45]$, respectively.

Lemma 2.2. 45 Suppose that $0<\alpha<1$, then the coefficients $\omega_{k}^{(\alpha)}$ satisfy

$$
\left\{\begin{array}{l}
\omega_{0}^{(\alpha)}=\frac{\alpha}{2}>0, \quad \omega_{1}^{(\alpha)}=\frac{2-\alpha-\alpha^{2}}{2}>0, \quad \omega_{2}^{(\alpha)}=\frac{\alpha\left(\alpha^{2}+\alpha-4\right)}{4}<0 \\
\omega_{2}^{(\alpha)}<\omega_{3}^{(\alpha)}<\omega_{4}^{(\alpha)}<\cdots<0, \quad \omega_{0}^{(\alpha)}+\omega_{2}^{(\alpha)}<0 \\
\sum_{k=0}^{\infty} \omega_{k}^{(\alpha)}=0, \quad \sum_{k=0}^{N} \omega_{k}^{(\alpha)}>0, \quad N \geq 1
\end{array}\right.
$$

Lemma 2.3. 45 Suppose that $1<\beta<2$, then the coefficients $\omega_{k}^{(\beta)}$ satisfy

$$
\left\{\begin{array}{l}
\omega_{0}^{(\beta)}=\frac{\beta}{2}>0, \quad \omega_{1}^{(\beta)}=\frac{2-\beta-\beta^{2}}{2}<0, \quad \omega_{2}^{(\beta)}=\frac{\beta\left(\beta^{2}+\beta-4\right)}{4} \\
1 \geq \omega_{0}^{(\beta)} \geq \omega_{3}^{(\beta)} \geq \omega_{4}^{(\beta)} \geq \cdots \geq 0, \quad \omega_{0}^{(\beta)}+\omega_{2}^{(\beta)}>0 \\
\sum_{k=0}^{\infty} \omega_{k}^{(\beta)}=0, \quad \sum_{k=0}^{N} \omega_{k}^{(\beta)}<0, \quad N \geq 2
\end{array}\right.
$$

In the rest of this paper, we define the discrete inner product and the corresponding discrete $L_{2}$-norm as follows,

$$
(\boldsymbol{u}, \boldsymbol{v})=h \sum_{i=1}^{N-1} u_{i} v_{i}, \quad\|\boldsymbol{u}\|=\sqrt{(\boldsymbol{u}, \boldsymbol{u})}, \quad \text { for } \forall \boldsymbol{u}, \boldsymbol{v} \in V_{h}
$$

where

$$
V_{h}=\left\{\boldsymbol{v} \mid \boldsymbol{v}=\left\{v_{i}\right\} \text { is a grid function on } \bar{\omega}_{h} \text { and } v_{i}=0 \text { if } i=0, N\right\} .
$$

Based on the above lemmas, we will provide some other lemmas and corollaries, which are important properties to proof the stability and the convergence of the implicit difference scheme (2.4).

Lemma 2.4. For $0<\alpha<1$, and any $\boldsymbol{v} \in V_{h}$, it holds that

$$
\left(\delta_{x,+}^{\alpha} \boldsymbol{v}, \boldsymbol{v}\right)=\left(\delta_{x,-}^{\alpha} \boldsymbol{v}, \boldsymbol{v}\right) \geq\left(\frac{1}{h^{\alpha}} \sum_{k=0}^{N-1} \omega_{k}^{(\alpha)}\right)\|\boldsymbol{v}\|^{2} .
$$

Proof. According to the discrete definitions of $\delta_{x,+}^{\alpha}$ and $\delta_{x,-}^{\alpha}$, and $v_{0}=v_{N}=0$, we have

$$
\left(\delta_{x,+}^{\alpha} \boldsymbol{v}, \boldsymbol{v}\right)=\left(\delta_{x,-}^{\alpha} \boldsymbol{v}, \boldsymbol{v}\right)
$$


and

$$
\begin{aligned}
\left(\delta_{x,+}^{\alpha} \boldsymbol{v}, \boldsymbol{v}\right)= & h^{1-\alpha} \sum_{i=1}^{N-1}\left(\sum_{k=0}^{i+1} \omega_{k}^{(\alpha)} v_{i-k+1}\right) v_{i} \\
= & \omega_{1}^{(\alpha)} h^{1-\alpha} \sum_{i=1}^{N-1}\left(v_{i}\right)^{2}+\left(\omega_{0}^{(\alpha)}+\omega_{2}^{(\alpha)}\right) h^{1-\alpha} \sum_{i=1}^{N-2} v_{i} v_{i+1}+\sum_{k=3}^{N-1} \omega_{k}^{(\alpha)} h^{1-\alpha} \sum_{i=1}^{N-k} v_{i+k-1} v_{i} \\
\geq & \omega_{1}^{(\alpha)} h^{-\alpha}\|\boldsymbol{v}\|^{2}+\left(\omega_{0}^{(\alpha)}+\omega_{2}^{(\alpha)}\right) h^{1-\alpha} \sum_{i=1}^{N-2} \frac{\left(v_{i}\right)^{2}+\left(v_{i+1}\right)^{2}}{2} \\
& +\sum_{k=3}^{N-1} \omega_{k}^{(\alpha)} h^{1-\alpha} \sum_{i=1}^{N-k} \frac{\left(v_{i+k-1}\right)^{2}+\left(v_{i}\right)^{2}}{2} \geq\left(\frac{1}{h^{\alpha}} \sum_{k=0}^{N-1} \omega_{k}^{(\alpha)}\right)\|\boldsymbol{v}\|^{2} .
\end{aligned}
$$

Further, the following conclusion is true.

Corollary 1. For $0<\alpha<1$, and any $\boldsymbol{v} \in V_{h}, N \geq 2$, there exists a positive constant $c_{1}$, such that

$$
\left(\delta_{x,+}^{\alpha} \boldsymbol{v}, \boldsymbol{v}\right)=\left(\delta_{x,-}^{\alpha} \boldsymbol{v}, \boldsymbol{v}\right)>c_{1} \ln 2\|\boldsymbol{v}\|^{2} .
$$

Proof. Since

$$
\sum_{k=N}^{2 N+2} \omega_{k}^{(\alpha)}=\sum_{k=N}^{2 N} g_{k}^{(\alpha)}+\frac{2-\alpha}{2} g_{N-1}^{(\alpha)}+\frac{\alpha}{2} g_{2 N+2}^{(\alpha)}+g_{2 N+1}^{(\alpha)} .
$$

According to the nature of $g_{k}^{(\alpha)}$ [1,2,45], the following inequality is established:

$$
\sum_{k=N}^{2 N+2} \omega_{k}^{(\alpha)}<\sum_{k=N}^{2 N} g_{k}^{(\alpha)}, \quad N \geq 2 .
$$

Then, there exist two positive constants $\tilde{c}_{1}$ and $c_{1}$, such that

$$
\begin{aligned}
\frac{1}{h^{\alpha}} \sum_{k=N}^{\infty}\left(-\omega_{k}^{(\alpha)}\right) & >\frac{1}{h^{\alpha}} \sum_{k=N}^{2 N+2}\left(-\omega_{k}^{(\alpha)}\right)>\frac{1}{h^{\alpha}} \sum_{k=N}^{2 N}\left(-g_{k}^{(\alpha)}\right) \\
& \geq \tilde{c}_{1} \sum_{k=N}^{2 N} k^{-(\alpha+1)} N^{\alpha}>\tilde{c}_{1} \sum_{k=N}^{2 N} k^{-(\alpha+1)}\left(\frac{k}{2}\right)^{\alpha} \\
& =\frac{\tilde{c}_{1}}{2^{\alpha}} \sum_{k=N}^{2 N} \frac{1}{k}>c_{1} \int_{N}^{2 N+1} \frac{1}{x} d x \geq c_{1} \int_{N}^{2 N} \frac{1}{x} d x=c_{1} \ln 2, \quad N \geq 2 .
\end{aligned}
$$

Here, the penultimate and antepenult inequalities are true due to the fact that $J_{1}(x)=1 / x$ is a lower convex function and $J_{2}(x)=\ln (x)>0, x \in[N, 2 N+1]$ is an increasing function.

Combining with Lemmas 2.2 and 2.4, we obtain 


$$
\left(\delta_{x,+}^{\alpha} \boldsymbol{v}, \boldsymbol{v}\right)=\left(\delta_{x,-}^{\alpha} \boldsymbol{v}, \boldsymbol{v}\right) \geq \frac{1}{h^{\alpha}} \sum_{k=N}^{\infty}\left(-\omega_{k}^{(\alpha)}\right)\|\boldsymbol{v}\|^{2}>c_{1} \ln 2\|\boldsymbol{v}\|^{2}
$$

Using the same argument as in the proof of Corollary 1, we can easily carry out the following corollary.

Corollary 2. (1) For $1<\beta<2$, and any $\boldsymbol{v} \in V_{h}$, it holds that

$$
\left(\delta_{x,+}^{\beta} \boldsymbol{v}, \boldsymbol{v}\right)=\left(\delta_{x,-}^{\beta} \boldsymbol{v}, \boldsymbol{v}\right) \leq\left(\frac{1}{h^{\beta}} \sum_{k=0}^{N-1} \omega_{k}^{(\beta)}\right)\|\boldsymbol{v}\|^{2} .
$$

(2) For $1<\beta<2$, and any $\boldsymbol{v} \in V_{h}, N \geq 3$, there exists a positive constant $c_{2}$, such that

$$
\left(-\delta_{x,+}^{\beta} \boldsymbol{v}, \boldsymbol{v}\right)=\left(-\delta_{x,-}^{\beta} \boldsymbol{v}, \boldsymbol{v}\right)>c_{2} \ln 2\|\boldsymbol{v}\|^{2} .
$$

With the help of the preceding lemmas and corollaries, we can now establish the following theorem, which is essential for analyzing the stability of the proposed implicit difference scheme.

Theorem 2.1. For any $\boldsymbol{v} \in V_{h}$, it holds that

$$
\left(\delta_{h}^{\alpha, \beta} \boldsymbol{v}, \boldsymbol{v}\right) \leq-c \ln 2\|\boldsymbol{v}\|^{2},
$$

where $c$ is a positive constant independent of the spatial step size $h$.

Proof. The concrete expression of $\left(\delta_{h}^{\alpha, \beta} \boldsymbol{v}, \boldsymbol{v}\right)$ can be written as

$$
\left(\delta_{h}^{\alpha, \beta} \boldsymbol{v}, \boldsymbol{v}\right)=-d_{+}^{j+\sigma}\left(\delta_{x,+}^{\alpha} \boldsymbol{v}, \boldsymbol{v}\right)-d_{-}^{j+\sigma}\left(\delta_{x,-}^{\alpha} \boldsymbol{v}, \boldsymbol{v}\right)+e_{+}^{j+\sigma}\left(\delta_{x,+}^{\beta} \boldsymbol{v}, \boldsymbol{v}\right)+e_{-}^{j+\sigma}\left(\delta_{x,-}^{\beta} \boldsymbol{v}, \boldsymbol{v}\right) .
$$

According to Corollaries 1,2, there exist two positive constants $c_{1}$ and $c_{2}$ independent of the spatial step size $h$, such that for any non-vanishing vector $\boldsymbol{v} \in V_{h}$, we have

$$
\begin{aligned}
\left(\delta_{h}^{\alpha, \beta} \boldsymbol{v}, \boldsymbol{v}\right) & =-d_{+}^{j+\sigma}\left(\delta_{x,+}^{\alpha} \boldsymbol{v}, \boldsymbol{v}\right)-d_{-}^{j+\sigma}\left(\delta_{x,-}^{\alpha} \boldsymbol{v}, \boldsymbol{v}\right)+e_{+}^{j+\sigma}\left(\delta_{x,+}^{\beta} \boldsymbol{v}, \boldsymbol{v}\right)+e_{-}^{j+\sigma}\left(\delta_{x,-}^{\beta} \boldsymbol{v}, \boldsymbol{v}\right) \\
& <-\ln 2\left[c_{1}\left(d_{+}^{j+\sigma}+d_{-}^{j+\sigma}\right)+c_{2}\left(e_{+}^{j+\sigma}+e_{-}^{j+\sigma}\right)\right]\|\boldsymbol{v}\|^{2} .
\end{aligned}
$$

For simplicity, we may take $c=c_{1}\left(d_{+}^{j+\sigma}+d_{-}^{j+\sigma}\right)+c_{2}\left(e_{+}^{j+\sigma}+e_{-}^{j+\sigma}\right)$. Hence, the targeted result is immediately completed.

Now we can proof the stability and convergence of the implicit difference scheme (2.4). For convenience, in our proof, we denote $q_{s}^{j+1}=\frac{\tau^{-\theta} c_{j-s}^{(\theta, \sigma)}}{\Gamma(2-\theta)}$. Then $\Delta_{0, t_{j+\sigma}}^{\theta}=\sum_{s=0}^{j}\left(u^{s+1}-u^{s}\right) q_{s}^{j+1}$.

Theorem 2.2. Denote $u^{j+1}=\left(u_{1}^{j+1}, u_{2}^{j+1}, \ldots, u_{N-1}^{j+1}\right)^{T}$ and $\left\|f^{j+\sigma}\right\|^{2}=h \sum_{i=1}^{N-1} f^{2}\left(x_{i}, t_{j+\sigma}\right)$. Then the implicit difference scheme 2.4] is unconditionally stable for $N \geq 3$, and the following a priori estimate holds:

$$
\left\|u^{j+1}\right\|^{2} \leq\left\|u^{0}\right\|^{2}+\frac{T^{\theta} \Gamma(1-\theta)}{c \ln 2}\left\|f^{j+\sigma}\right\|^{2}, \quad 0 \leq j \leq M-1 .
$$


Proof. Taking the inner product of (2.4) with $u^{(j+\sigma)}$, we have

$$
\left(\Delta_{0, t_{j+\sigma}}^{\theta} u, u^{(j+\sigma)}\right)=\left(\delta_{h}^{\alpha, \beta} u^{(j+\sigma)}, u^{(j+\sigma)}\right)+\left(f^{j+\sigma}, u^{(j+\sigma)}\right) .
$$

Using Corollary 1 in [43] and Theorem 2.1, obtains

$$
\frac{1}{2} \Delta_{0, t_{j+\sigma}}^{\theta}\|u\|^{2} \leq-c \ln 2\left\|u^{(j+\sigma)}\right\|^{2}+\varepsilon\left\|u^{(j+\sigma)}\right\|^{2}+\frac{1}{4 \varepsilon}\left\|f^{j+\sigma}\right\|^{2}, \quad \varepsilon>0 .
$$

From (2.6), at $\varepsilon=c \ln 2$ we get

$$
\frac{1}{2} \Delta_{0, t_{j+\sigma}}^{\theta}\|u\|^{2} \leq \frac{1}{4 c \ln 2}\left\|f^{j+\sigma}\right\|^{2} .
$$

Let us rewrite inequality (2.7) in the form

$$
q_{j}^{j+1}\left\|u^{j+1}\right\|^{2} \leq \sum_{s=1}^{j}\left(q_{s}^{j+1}-q_{s-1}^{j+1}\right)\left\|u^{s}\right\|^{2}+q_{0}^{j+1}\left(\left\|u^{0}\right\|^{2}+\frac{T^{\theta} \Gamma(1-\theta)}{c \ln 2}\left\|f^{j+\sigma}\right\|^{2}\right),
$$

noticing that $q_{0}^{j+1}>\frac{1}{2 T^{\theta} \Gamma(1-\theta)}$ (cf. [47]).

With the inequality (2.8) in hand, the inequality (2.5) can be reached through utilizing the mathematical induction method.

The proof of Theorem 2.2 is completed.

With the above proof, the convergence of the implicit difference scheme (2.4) is easy to obtain.

Theorem 2.3. Suppose that $u(x, t)$ is the solution of (1.1) and $\left\{u_{i}^{j} \mid x_{i} \in \bar{\omega}_{h}, \quad 0 \leq j \leq M\right\}$ is the solution of the implicit difference scheme (2.4). Denote

$$
\xi_{i}^{j}=u\left(x_{i}, t_{j}\right)-u_{i}^{j}, \quad x_{i} \in \bar{\omega}_{h}, \quad 0 \leq j \leq M .
$$

Then there exists a positive constant $\tilde{c}$ such that

$$
\left\|\xi^{j}\right\| \leq \tilde{c}\left(\tau^{2}+h^{2}\right), \quad 0 \leq j \leq M
$$

Proof. It can easily obtain that $\xi^{j}$ satisfies the following error equation

$$
\begin{cases}\Delta_{0, t_{j+\sigma}}^{\theta} \xi_{i}=\delta_{h}^{\alpha, \theta} \xi_{i}^{(j+\sigma)}+R_{i}^{j+\sigma}, & 1 \leq i \leq N-1, \quad 0 \leq j \leq M-1, \\ \xi_{i}^{0}=0, & 1 \leq i \leq N-1, \\ \xi_{0}^{j}=\xi_{N}^{j}=0, & 0 \leq j \leq M,\end{cases}
$$

where $R_{i}^{j+\sigma}=\mathcal{O}\left(\tau^{2}+h^{2}\right)$. By exploiting Theorem 2.2 we get

$$
\left\|\xi^{j+1}\right\|^{2} \leq \frac{T^{\theta} \Gamma(1-\theta)}{c \ln 2}\left\|R^{j+\sigma}\right\|^{2} \leq \tilde{c}\left(\tau^{2}+h^{2}\right), \quad 0 \leq j \leq M-1,
$$

which implies the convergence in the mesh $L_{2}$-norm with rate $\mathcal{O}\left(\tau^{2}+h^{2}\right)$.

The unconditionally stability and convergence of IDS (2.4) have been proved, and a numerical test is displayed to verify our results in Section 4. 


\subsection{A nonlinear extension of TSFADE}

In this subsection, we extend the TSFADE to nonlinear case:

$$
\left\{\begin{aligned}
D_{0, t}^{\theta} u(x, t)= & -d_{+}(t) D_{0, x}^{\alpha} u(x, t)-d_{-}(t) D_{x, L}^{\alpha} u(x, t) \\
\quad+e_{+}(t) D_{0, x}^{\beta} u(x, t)+e_{-}(t) D_{x, L}^{\beta} u(x, t)+g(u, x, t), & 0 \leq x \leq L, \\
u(x, 0)=u_{0}(x), & 0 \leq t \leq T, \\
u(0, t)=u(L, t)=0, &
\end{aligned}\right.
$$

where $g(u(x, t), x, t)=f(x, t)+y(u(x, t))$.

Considering (2.9) at point $\left(x_{i}, t_{j+\sigma}\right)$ and doing some simple manipulations, we have

$$
\begin{cases}\Delta_{0, t_{j+\sigma}}^{\theta} u_{i}=\delta_{h}^{\alpha, \beta} u_{i}^{(\sigma)}+g_{i}^{j+\sigma}, & 1 \leq i \leq N-1, \quad 0 \leq j \leq M-1, \\ u_{i}^{0}=u_{0}\left(x_{i}\right), & 1 \leq i \leq N-1, \\ u_{0}^{j}=u_{N}^{j}=0, & 0 \leq j \leq M-1,\end{cases}
$$

in which $g_{i}^{j+\sigma}=g\left(\sigma u_{i}^{j+1}+(1-\sigma) u_{i}^{j}, x_{i}, t_{j+\sigma}\right)$, and the truncation error is $\mathcal{O}\left(\tau^{2}+h^{2}\right)$.

We assume $U_{i}^{j}$ is the approximation solution of $u_{i}^{j}$ given in (2.10), and let $\xi_{i}^{j}=u_{i}^{j}-U_{i}^{j}, i=$ $1, \cdots, N-1 ; j=1, \cdots, M-1$, be the error satisfying

$$
\Delta_{0, t_{j+\sigma}}^{\theta} \zeta_{i}=\delta_{h}^{\alpha, \beta} \zeta_{i}^{(\sigma)}+y\left(u_{i}^{j+\sigma}\right)-y\left(U_{i}^{j+\sigma}\right)
$$

Correspondingly, we denote $E^{j}=\left[\zeta_{1}^{j}, \zeta_{2}^{j}, \cdots, \zeta_{N-1}^{j}\right]^{T}(j=1, \cdots, M-1)$, and suppose the nonlinear term $y(u(x, t))$ satisfies:

$$
|y(u(x, t))-y(v(x, t))| \leq L_{1}|u(x, t)-v(x, t)|, \text { for all } u(x, t), v(x, t) \text { over }[0, L] \times[0, T]
$$

with $0<L_{1}<\frac{a_{0}^{(\theta, \sigma)}}{2\left[\sigma^{2}+(1-\sigma)^{2}\right] \tau^{2} \Gamma(2-\theta)}$. Now, we proof the stability of (2.10) .

Theorem 2.4. The implicit difference scheme (2.10) is stable, i.e.:

$$
\left\|E^{j+1}\right\| \leq \tilde{C}\left\|E^{0}\right\|, j=0,1, \cdots, M-1,
$$

where $\tilde{C}$ is a positive constant, which may independent of $h$.

Proof. Taking the inner product of (2.11) with $E^{(j+\sigma)}$, we have

$$
\left(\Delta_{0, t_{j+\sigma}}^{\theta} E, E^{(j+\sigma)}\right)=\left(\delta_{h}^{\alpha, \beta} E^{(j+\sigma)}, E^{(j+\sigma)}\right)+\left(y\left(u^{j+\sigma}\right)-y\left(U^{j+\sigma}\right), E^{(j+\sigma)}\right) .
$$

Utilizing Corollary 1 in [43] and noticing $\left(\delta_{h}^{\alpha, \beta} E^{(j+\sigma)}, E^{(j+\sigma)}\right)<0$, gets

$$
\frac{1}{2} \Delta_{0, t_{j+\sigma}}^{\theta}\|E\|^{2} \leq L_{1}\left\|E^{(j+\sigma)}\right\|^{2} .
$$

The right hand side can be estimated as below

$$
\begin{aligned}
\left\|E^{(j+\sigma)}\right\|^{2} & =\sigma^{2}\left\|E^{j+1}\right\|^{2}+(1-\sigma)^{2}\left\|E^{j}\right\|^{2}+2\left(\sigma E^{j+1},(1-\sigma) E^{j}\right) \\
& =\sigma^{2}\left\|E^{j+1}\right\|^{2}+(1-\sigma)^{2}\left\|E^{j}\right\|^{2}+2\left((1-\sigma) E^{j+1}, \sigma E^{j}\right) \\
& \leq\left[\sigma^{2}+(1-\sigma)^{2}\right]\left(\left\|E^{j+1}\right\|^{2}+\left\|E^{j}\right\|^{2}\right) .
\end{aligned}
$$


Bringing the above estimate to inequality (2.12), obtains

$$
\begin{gathered}
\left\{\frac{c_{0}^{(\theta, \sigma)}}{2 \tau^{\theta} \Gamma(2-\theta)}-L_{1}\left[\sigma^{2}+(1-\sigma)^{2}\right]\right\}\left\|E^{j+1}\right\|^{2} \\
\leq L_{1}\left[\sigma^{2}+(1-\sigma)^{2}\right]\left\|E^{j}\right\|^{2}+\frac{c_{j}^{(\theta, \sigma)}}{2 \tau^{\theta} \Gamma(2-\theta)}\left\|E^{0}\right\|^{2} \\
\quad+\frac{1}{2 \tau^{\theta} \Gamma(2-\theta)} \sum_{s=1}^{j}\left(c_{j-s}^{(\theta, \sigma)}-c_{j-s+1}^{(\theta, \sigma)}\right)\left\|E^{s}\right\|^{2} .
\end{gathered}
$$

Employing the mathematical induction method, the targeted result is immediately completed.

Next, we study the convergence of (2.10).

Theorem 2.5. Suppose that $u(x, t)$ is the solution of (2.9) and $\left\{u_{i}^{j} \mid x_{i} \in \bar{\omega}_{h}, \quad 0 \leq j \leq M\right\}$ is the solution of the implicit difference scheme (2.10). Denote

$$
\psi_{i}^{j}=u\left(x_{i}, t_{j}\right)-u_{i}^{j}, \quad x_{i} \in \bar{\omega}_{h}, \quad 0 \leq j \leq M .
$$

Then there exists a positive constant $\hat{C}$ such that

$$
\left\|\psi^{j}\right\| \leq \hat{C}\left(\tau^{2}+h^{2}\right), \quad 0 \leq j \leq M .
$$

Proof. Subtract (2.10) from (2.9), obtains

$$
\begin{cases}\Delta_{0, t_{j+\sigma}}^{\theta} \psi_{i}=\delta_{h}^{\alpha, \beta} \psi_{i}^{(\sigma)}+y\left(u\left(x_{i}, t_{j+\sigma}\right)\right)-y\left(u_{i}^{j+\sigma}\right)+\tilde{R}_{i}^{j+\sigma}, & 1 \leq i \leq N-1, \quad 0 \leq j \leq M-1, \\ \psi_{i}^{0}=0, & 1 \leq i \leq N-1, \\ \psi_{0}^{j}=\psi_{N}^{j}=0, & 0 \leq j \leq M-1,\end{cases}
$$

where $\tilde{R}_{i}^{j+\sigma}=\mathcal{O}\left(\tau^{2}+h^{2}\right)$. For convenience, we assume that there is a positive constant $\bar{C}$ such that: $\tilde{R}_{i}^{j+\sigma} \leq \bar{C}\left(\tau^{2}+h^{2}\right), 1 \leq i \leq N-1,0 \leq j \leq M-1$.

Taking inner product of this error equation with $\psi^{j+\sigma}$, we have

$$
\left(\Delta_{0, t_{j+\sigma}}^{\theta} \psi, \psi^{(j+\sigma)}\right)=\left(\delta_{h}^{\alpha, \beta} \psi^{(j+\sigma)}, \psi^{(j+\sigma)}\right)+\left(y\left(u^{j+\sigma}\right)-y\left(U^{j+\sigma}\right), \psi^{(j+\sigma)}\right)+\left(\tilde{R}_{i}^{j+\sigma}, \psi^{(j+\sigma)}\right) .
$$

Similar as the proof of Theorem 2.2, we arrive at

$$
\begin{gathered}
\left\{\frac{c_{0}^{(\theta, \sigma)}}{2 \tau^{\theta} \Gamma(2-\theta)}-L_{1}\left[\sigma^{2}+(1-\sigma)^{2}\right]\right\}\left\|\psi^{j+1}\right\|^{2} \\
\leq L_{1}\left[\sigma^{2}+(1-\sigma)^{2}\right]\left\|\psi^{j}\right\|^{2}+\frac{1}{4 c \ln 2}\left\|\tilde{R}^{j+\sigma}\right\|^{2} \\
\quad+\frac{1}{2 \tau^{\theta} \Gamma(2-\theta)} \sum_{s=1}^{j}\left(c_{j-s}^{(\theta, \sigma)}-c_{j-s+1}^{(\theta, \sigma)}\right)\left\|\psi^{s}\right\|^{2} .
\end{gathered}
$$

Utilizing the mathematical induction method, the priori estimate follows that

$$
\left\|\psi^{j}\right\| \leq \hat{C}\left(\tau^{2}+h^{2}\right), \quad 0 \leq j \leq M .
$$

The proof of this theorem is completed. 
The two assumptions about $y(u(x, t))$ are extremely strict, which is not very satisfactory. So our further work is to seek a more suitable method to prove the stability of IDS (2.10).

\section{Fast implementation of IDS with the circulant precondtioner}

Before moving into the investigation of fast solution techniques, the matrix form of implicit difference scheme (2.4) must be established first. In order to facilitate our discussion, we use notations in Section 2, Then, the matrix form of (2.4) corresponding to each time layer $j$ can be written as follows:

$$
\mathcal{M}^{j+\sigma} u^{j+1}=B^{j+\sigma} u^{j}-\frac{\tau^{-\theta}}{\Gamma(2-\theta)} \sum_{s=0}^{j-1} c_{j-s}^{(\theta, \sigma)}\left(u^{s+1}-u^{s}\right)+f^{j+\sigma}, \quad 0 \leq j \leq M-1,
$$

we assume that the sums to be equal to zero if the upper summation index is less than the lower one, where

$$
\begin{gathered}
\mathcal{M}^{j+\sigma}=\frac{\tau^{-\theta}}{\Gamma(2-\theta)} c_{0}^{(\theta, \sigma)} I+\sigma\left(\frac{d_{+}^{j+\sigma}}{h^{\alpha}} A_{\alpha}+\frac{d_{-}^{j+\sigma}}{h^{\alpha}} A_{\alpha}^{T}-\frac{e_{+}^{j+\sigma}}{h^{\beta}} A_{\beta}-\frac{e_{-}^{j+\sigma}}{h^{\beta}} A_{\beta}^{T}\right), \\
B^{j+\sigma}=\frac{\tau^{-\theta}}{\Gamma(2-\theta)} c_{0}^{(\theta, \sigma)} I-(1-\sigma)\left(\frac{d_{+}^{j+\sigma}}{h^{\alpha}} A_{\alpha}+\frac{d_{-}^{j+\sigma}}{h^{\alpha}} A_{\alpha}^{T}-\frac{e_{+}^{j+\sigma}}{h^{\beta}} A_{\beta}-\frac{e_{-}^{j+\sigma}}{h^{\beta}} A_{\beta}^{T}\right),
\end{gathered}
$$

and $I$ is an identity matrix of order $N-1$, the two matrices $A_{\alpha}$ and $A_{\beta}$ are defined by

$$
A_{\alpha}=\left[\begin{array}{ccccc}
\omega_{1}^{(\alpha)} & \omega_{0}^{(\alpha)} & & & \\
\omega_{2}^{(\alpha)} & \omega_{1}^{(\alpha)} & \omega_{0}^{(\alpha)} & & \\
\vdots & \ddots & \ddots & \ddots & \\
\omega_{N-2}^{(\alpha)} & \ldots & \ddots & \ddots & \omega_{0}^{(\alpha)} \\
\omega_{N-1}^{(\alpha)} & \omega_{N-2}^{(\alpha)} & \cdots & \omega_{2}^{(\alpha)} & \omega_{1}^{(\alpha)}
\end{array}\right], \quad A_{\beta}=\left[\begin{array}{ccccc}
\omega_{1}^{(\beta)} & \omega_{0}^{(\beta)} & & & \\
\omega_{2}^{(\beta)} & \omega_{1}^{(\beta)} & \omega_{0}^{(\beta)} & & \\
\vdots & \ddots & \ddots & \ddots & \\
\omega_{N-2}^{(\beta)} & \cdots & \ddots & \ddots & \omega_{0}^{(\beta)} \\
\omega_{N-1}^{(\beta)} & \omega_{N-2}^{(\beta)} & \cdots & \omega_{2}^{(\beta)} & \omega_{1}^{(\beta)}
\end{array}\right]
$$

It is apparent that $A_{\alpha}$ and $A_{\beta}$ are Toeplitz matrices. Therefore, they can be stored with $2 N+2$ entries. Krylov subspace methods with suitable circulant preconditioners [37,48] can be used to efficiently solve Toeplitz or Toeplitz-like linear systems with a fast convergence rate. In this case, it also remarked that the algorithmic complexity of preconditioned Krylov subspace methods is only in $\mathcal{O}(N \log N)$ arithmetic operations per iteration step.

Inspired by the above consideration, we propose a circulant preconditioner to solve (3.1), which is generated from the Strang's circulant preconditioner [49], through two preconditioned Krylov subspace methods. The Strang's circulant matrix $s(Q)=\left[s_{j-k}\right]_{0 \leq j, k<N}$ for a real Toeplitz matrix $Q=\left[q_{j-k}\right]_{0 \leq j, k<N}$ is obtained by copying the central diagonals of $Q$ and bringing them around to complete the circulant requirement. More precisely, the diagonals of $s(Q)$ are given by

$$
s_{j}= \begin{cases}q_{j}, & 0 \leq j<N / 2, \\ 0, & j=N / 2 \text { if } N \text { is even }, \\ q_{j-N}, & N / 2<j<N, \\ s_{j+N}, & 0<-j<N .\end{cases}
$$


Then our circulant preconditioner is defined as

$$
P^{(j+\sigma)}=\left\{\begin{array}{c}
\frac{\tau^{-\theta}}{\Gamma(2-\theta)} a_{0}^{(\theta, \sigma)} I+\sigma\left(\frac{d_{+}^{j+\sigma}}{h^{\alpha}} s\left(A_{\alpha}\right)+\frac{d_{-}^{j+\sigma}}{h^{\alpha}} s\left(A_{\alpha}^{T}\right)\right. \\
\left.-\frac{e_{+}^{j+\sigma}}{h^{\beta}} s\left(A_{\beta}\right)-\frac{e_{-}^{j+\sigma}}{h^{\beta}} s\left(A_{\beta}^{T}\right)\right), \quad j=0, \\
\frac{\tau^{-\theta}}{\Gamma(2-\theta)}\left(a_{0}^{(\theta, \sigma)}+b_{1}^{(\theta, \sigma)}\right) I+\sigma\left(\frac{d_{+}^{j+\sigma}}{h^{\alpha}} s\left(A_{\alpha}\right)+\frac{d_{-}^{j+\sigma}}{h^{\alpha}} s\left(A_{\alpha}^{T}\right)\right. \\
\left.-\frac{e_{+}^{j+\sigma}}{h^{\beta}} s\left(A_{\beta}\right)-\frac{e_{-}^{j+\sigma}}{h^{\beta}} s\left(A_{\beta}^{T}\right)\right), \quad j=1, \cdots, M-1,
\end{array}\right.
$$

where the first columns of $s\left(A_{\gamma}\right)$ and $s\left(A_{\gamma}^{T}\right)(\gamma=\alpha, \beta)$ are given by

$$
\left[\begin{array}{c}
\omega_{1}^{(\gamma)} \\
\vdots \\
\omega_{\left\lfloor\frac{N}{2}\right\rfloor}^{(\gamma)} \\
0 \\
\vdots \\
0 \\
\omega_{0}^{(\gamma)}
\end{array}\right] \text { and }\left[\begin{array}{c}
\omega_{1}^{(\gamma)} \\
\omega_{0}^{(\gamma)} \\
0 \\
\vdots \\
0 \\
\omega_{\left\lfloor\frac{N}{2}\right\rfloor}^{(\gamma)} \\
\vdots \\
\omega_{2}^{(\gamma)}
\end{array}\right] \text {, respectively. }
$$

To make sure the preconditioner defined in (3.2) is well-defined, let us illustrate that $P^{(j+\sigma)}$ are nonsingular. Before that, we need to give the following theorem, which is essential to check the nonsingularity of $P^{(j+\sigma)}$.

Theorem 3.1. 1. The real parts of all eigenvalues of $s\left(A_{\alpha}\right)$ and $s\left(A_{\alpha}^{T}\right)$ are strictly positive for all $N$;

2. The real parts of all eigenvalues of $s\left(A_{\beta}\right)$ and $s\left(A_{\beta}^{T}\right)$ are strictly negative for all $N$.

Proof. Firstly, we proof the real parts of all eigenvalues of $s\left(A_{\alpha}\right)$ are strictly positive for all $N$.

Recall that the real parts of all eigenvalues of $s\left(A_{\alpha}\right)$ are equivalent to the eigenvalues of $\frac{s\left(A_{\alpha}\right)+\left[s\left(A_{\alpha}\right)\right]^{*}}{2}$ (where $S^{*}$ represents conjugate transpose of matrix $S$ ). Therefore, it is sufficient to show that all eigenvalues of $\frac{s\left(A_{\alpha}\right)+s^{*}\left(A_{\alpha}\right)}{2}$ are strictly positive for all $N$.

According to the Gershgorin circle theorem, all the Gershgorin disc of the circulant matrix $\frac{s\left(A_{\alpha}\right)+s^{*}\left(A_{\alpha}\right)}{2}$ are centered at $\omega_{1}^{(\alpha)}>0$ with radius

$$
r_{\alpha}=-\sum_{k=0, k \neq 1}^{\left\lfloor\frac{N}{2}\right\rfloor} \omega_{k}^{(\alpha)}<-\sum_{k=0, k \neq 1}^{\infty} \omega_{k}^{(\alpha)}=\omega_{1}^{(\alpha)},
$$

by the Lemma 2.2, the first part of this theorem is proved.

Then, we prove the second part of the theorem. Similarly, all the Gershgorin disc of the circulant matrix $\frac{s\left(A_{\beta}\right)+s^{*}\left(A_{\beta}\right)}{2}$ are centered at $\omega_{1}^{(\beta)}<0$ with radius

$$
r_{\beta}=\sum_{k=0, k \neq 1}^{\left\lfloor\frac{N}{2}\right\rfloor} \omega_{k}^{(\beta)}<\sum_{k=0, k \neq 1}^{\infty} \omega_{k}^{(\beta)}=-\omega_{1}^{(\beta)},
$$


by the Lemma 2.3, the second part of this theorem is proved. Finally, we complete the proof of the theorem.

With the help of Theorem 3.1. we will check the circulant preconditioners $P^{(j+\sigma)}$ are nonsingular.

Theorem 3.2. The circulant preconditioners $P^{(j+\sigma)}$ defined in (3.2) are nonsingular.

Proof. As we know, a circulant matrix can be diagonalized by the Fourier matrix $\mathrm{F}$ [4]. Then it follows that $s\left(A_{\gamma}\right)=F^{*} \Lambda_{\gamma} F, s\left(A_{\gamma}^{T}\right)=F^{*} \bar{\Lambda}_{\gamma} F$, where $\bar{\Lambda}_{\gamma}$ is the complex conjugate of $\Lambda_{\gamma}$. Decompose the circulant matrix $P^{(j+\sigma)}=F^{*} \Lambda_{P} F$ with the diagonal matrix

$$
\Lambda_{P}=\left\{\begin{aligned}
& \frac{\tau^{-\theta}}{\Gamma(2-\theta)} a_{0}^{(\theta, \sigma)} I+\sigma\left(\frac{d_{+}^{j+\sigma}}{h^{\alpha}} \Lambda_{\alpha}+\frac{d_{-}^{j+\sigma}}{h^{\alpha}} \bar{\Lambda}_{\alpha}\right. \\
&\left.-\frac{e_{+}^{j+\sigma}}{h^{\beta}} \Lambda_{\beta}-\frac{e_{-}^{j+\sigma}}{h^{\beta}} \bar{\Lambda}_{\beta}\right), \quad j=0 \\
& \frac{\tau^{-\theta}}{\Gamma(2-\theta)}\left(a_{0}^{(\theta, \sigma)}+b_{1}^{(\theta, \sigma)}\right) I+\sigma\left(\frac{d_{+}^{j+\sigma}}{h^{\alpha}} \Lambda_{\alpha}+\frac{d_{-}^{j+\sigma}}{h^{\alpha}} \bar{\Lambda}_{\alpha}\right. \\
&\left.\quad-\frac{e_{+}^{j+\sigma}}{h^{\beta}} \Lambda_{\beta}-\frac{e_{-}^{j+\sigma}}{h^{\beta}} \bar{\Lambda}_{\beta}\right), \quad j=1, \cdots, M-1
\end{aligned}\right.
$$

Then the real part of $\Lambda_{P}$ can be written as

$$
\operatorname{Re}\left(\left[\Lambda_{P}\right]_{k, k}\right)=\left\{\begin{array}{c}
\frac{\tau^{-\theta}}{\Gamma(2-\theta)} a_{0}^{(\theta, \sigma)}+\sigma\left(\frac{d_{+}^{j+\sigma}}{h^{\alpha}} \operatorname{Re}\left(\left[\Lambda_{\alpha}\right]_{k, k}\right)+\frac{d_{-}^{j+\sigma}}{h^{\alpha}} \operatorname{Re}\left(\left[\bar{\Lambda}_{\alpha}\right]_{k, k}\right)\right. \\
\left.-\frac{e_{+}^{j+\sigma}}{h^{\beta}} \operatorname{Re}\left(\left[\Lambda_{\beta}\right]_{k, k}\right)-\frac{e_{-}^{j+\sigma}}{h^{\beta}} \operatorname{Re}\left(\left[\bar{\Lambda}_{\beta}\right]_{k, k}\right)\right), \quad j=0, \\
\frac{\tau^{-\theta}}{\Gamma(2-\theta)}\left(a_{0}^{(\theta, \sigma)}+b_{1}^{(\theta, \sigma)}\right)+\sigma\left(\frac{d_{+}^{j+\sigma}}{h^{\alpha}} \operatorname{Re}\left(\left[\Lambda_{\alpha}\right]_{k, k}\right)+\frac{d_{-}^{j+\sigma}}{h^{\alpha}} \operatorname{Re}\left(\left[\bar{\Lambda}_{\alpha}\right]_{k, k}\right)\right. \\
\left.-\frac{e_{+}^{j+\sigma}}{h^{\beta}} \operatorname{Re}\left(\left[\Lambda_{\beta}\right]_{k, k}\right)-\frac{e_{-}^{j+\sigma}}{h^{\beta}} \operatorname{Re}\left(\left[\bar{\Lambda}_{\beta}\right]_{k, k}\right)\right), \quad j=1, \cdots, M-1 .
\end{array}\right.
$$

Combining Theorem 3.1, we obtain

$$
\operatorname{Re}\left(\left[\Lambda_{P}\right]_{k, k}\right)>0
$$

Consequently, $P^{(j+\sigma)}$ are invertible.

Unfortunately, due to the properties of coefficients $\omega_{k}^{(\alpha)}$ and $\omega_{k}^{(\beta)}$, it is difficult to theoretically investigate the eigenvalue distributions of preconditioned matrix $\left(P^{(j+\sigma)}\right)^{-1} \mathcal{M}^{j+\sigma}$, but we still can give some figures to illustrate the clustering eigenvalue distributions of several specified preconditioned matrices in section 4 .

At the end of this section, we study the fast implementation of IDS (2.10). For convenience, (2.10) is recast as

$$
\mathcal{M}^{j+\sigma} u^{j+1}=B^{j+\sigma} u^{j}-\frac{\tau^{-\theta}}{\Gamma(2-\theta)} \sum_{s=0}^{j-1} c_{j-s}^{(\theta, \sigma)}\left(u^{s+1}-u^{s}\right)+F^{j+1}, \quad 0 \leq j \leq M-1,
$$


in which

$$
F^{j+1}=\left[g\left(\sigma u_{1}^{j+1}+(1-\sigma) u_{1}^{j}, x_{1}, t_{j+\sigma}\right), \cdots, g\left(\sigma u_{N-1}^{j+1}+(1-\sigma) u_{N-1}^{j}, x_{N-1}, t_{j+\sigma}\right)\right]^{T} .
$$

Then the following algorithm is utilized to solve (3.3),

$$
\begin{array}{r}
\mathcal{M}^{j+\sigma} u^{j+1(l+1)}=B^{j+\sigma} u^{j}-\frac{\tau^{-\theta}}{\Gamma(2-\theta)} \sum_{s=0}^{j-1} c_{j-s}^{(\theta, \sigma)}\left(u^{s+1}-u^{s}\right)+F^{j+1(l)}, \\
0 \leq j \leq M-1, l=0,1,2, \cdots
\end{array}
$$

with

$$
u_{0}^{j(l)}=u_{N}^{j(l)}=0, \quad u^{j+1(0)}= \begin{cases}u^{j}, & j=0 \\ 2 u^{j}-u^{j-1}, & j \geq 1\end{cases}
$$

and

$$
\begin{aligned}
F^{j+1(l)}=[ & g\left(\sigma u_{1}^{j+1(l)}+(1-\sigma) u_{1}^{j}, x_{1}, t_{j+\sigma}\right), \\
& \left.\quad \cdots, g\left(\sigma u_{N-1}^{j+1(l)}+(1-\sigma) u_{N-1}^{j}, x_{N-1}, t_{j+\sigma}\right)\right]^{T} .
\end{aligned}
$$

At each iteration, the above system is linearized, and compared with (3.1), the system has not changed significantly. Thus, the previously mentioned fast method can be applied directly to this system. In the next section, we provide two experiments to test the effectiveness of our fast method, even for nonlinear situation.

\section{Numerical results}

In this section, we carry out several numerical experiments, which are given in Examples $1-2$, to illustrate that our proposed IDM can indeed convergent with second order accuracy in both space and time. Some other numerical experiments, given in Examples 3-4, are reported to illustrate the effectiveness of the fast solution techniques. For direct solver, we choose LU factorization of MATLAB in Examples 3-4, and its CPU time is represented. For two Krylov subspace methods $(\operatorname{BiCGSTAB}$ and $\operatorname{GPBiCOR}(m, \ell))$ with circulant preconditioners, number of iterations required for convergence and CPU time of those methods are reported. We denote $\operatorname{PBiCGSTAB}$ and $\operatorname{PGPBiCOR}(m, \ell)$ as the preconditioned version of $\operatorname{BiCGSTAB}$ and $\operatorname{GPBiCOR}(m, \ell)$ respectively. The stopping criterion of those methods is

$$
\frac{\left\|r^{(k)}\right\|_{2}}{\left\|r^{(0)}\right\|_{2}}<10^{-12}
$$

where $r^{(k)}$ is the residual vector of the linear system after $k$ iterations, and the initial guess at each time step is chosen as the zero vector. All experiments were performed on a Windows 7 (32 bit) PC-Intel(R) Core(TM) i3-2130 CPU 3.40GHz, 4GB of RAM using MATLAB R2015b. 


\subsection{Verification of convergence rate}

Example 1. We consider the linear problem (1.1), take coefficients

$$
\begin{aligned}
& d_{+}(t)=\exp (t), \quad d_{-}(t)=3 \exp (-t), \\
& e_{+}(t)=(1+t)^{2}, \quad e_{-}(t)=1+t^{2},
\end{aligned}
$$

and the source term

$$
\begin{aligned}
f(x, t)= & \frac{\Gamma(3+\theta)}{\Gamma(3)} t^{2} x^{2}(1-x)^{2}+t^{\theta+2}\left\{\frac{\Gamma(3)}{\Gamma(3-\alpha)}\left[d_{+}(t) x^{2-\alpha}+d_{-}(t)(1-x)^{2-\alpha}\right]\right. \\
& -\frac{2 \Gamma(4)}{\Gamma(4-\alpha)}\left[d_{+}(t) x^{3-\alpha}+d_{-}(t)(1-x)^{3-\alpha}\right]+\frac{\Gamma(5)}{\Gamma(5-\alpha)}\left[d_{+}(t) x^{4-\alpha}+d_{-}(t)(1-x)^{4-\alpha}\right] \\
& -\frac{\Gamma(3)}{\Gamma(3-\beta)}\left[e_{+}(t) x^{2-\beta}+e_{-}(t)(1-x)^{2-\beta}\right]+\frac{2 \Gamma(4)}{\Gamma(4-\beta)}\left[e_{+}(t) x^{3-\beta}+e_{-}(t)(1-x)^{3-\beta}\right] \\
& \left.-\frac{\Gamma(5)}{\Gamma(5-\beta)}\left[e_{+}(t) x^{4-\beta}+e_{-}(t)(1-x)^{4-\beta}\right]\right\} .
\end{aligned}
$$

Then the causal solution is $u(x, t)=t^{\theta+2} x^{2}(1-x)^{2}$.

As can be seen in Tables 1 and 3 , when $h=1 / 3000$, the maximum error decreases steadily with the shortening of time step, and the convergence order of time is the expected $\mathcal{O}\left(\tau^{2}\right)$, where the convergence order $(\mathrm{CO})$ is given by the following formula: $\mathrm{CO}=\log _{\tau_{1} / \tau_{2}} \frac{\left\|\xi_{1}\right\|}{\left\|\xi_{2}\right\|}$ ( $\xi_{i}$ is the error corresponding to $h_{i}$ ). On the other hand, Tables 2 and 4 illustrate that if $h=\tau$, a reduction in the maximum error occurs along with the decrease of space step and time step, and the spatial convergence order is $\mathcal{O}\left(h^{2}\right)$, where the convergence order (CO) is given by the formula: $\mathrm{CO}=\log _{\mathrm{h}_{1} / \mathrm{h}_{2}}\left\|\xi_{1}\right\|$. Figs. 1 $1 / 2$ are plotted to further explain the reliability of our proposed scheme, and indicate that the 'quadratic-type' order of accuracy can achieve the desired order $\mathcal{O}\left(\tau^{2}+h^{2}\right)$. 
Table 1: $L_{2}$-norm and maximum norm error behavior versus $\tau$-grid size reduction when $\alpha=0.6$, $\beta=1.8$, and $h=1 / 3000$ in Example 1 .

\begin{tabular}{cccccc}
\hline$\theta$ & $\tau$ & $\|\xi\|_{\mathcal{C}\left(\bar{\omega}_{h \tau}\right)}$ & CO in $\|\cdot\|_{\mathcal{C}\left(\bar{\omega}_{h \tau}\right)}$ & $\max _{0 \leq n \leq M}\left\|\xi^{n}\right\|_{0}$ & CO in $\|\cdot\|_{0}$ \\
\hline 0.10 & $1 / 8$ & $5.2321 \mathrm{e}-05$ & - & $3.3293 \mathrm{e}-05$ & - \\
& $1 / 16$ & $1.3096 \mathrm{e}-05$ & 1.9982 & $8.3340 \mathrm{e}-06$ & 1.9981 \\
& $1 / 32$ & $3.2603 \mathrm{e}-06$ & 2.0061 & $2.0748 \mathrm{e}-06$ & 2.0061 \\
& $1 / 64$ & $7.9868 \mathrm{e}-07$ & 2.0293 & $5.0828 \mathrm{e}-07$ & 2.0292 \\
& $1 / 128$ & $1.8626 \mathrm{e}-07$ & 2.1003 & $1.1850 \mathrm{e}-07$ & 2.1007 \\
\hline 0.50 & $1 / 8$ & $3.3405 \mathrm{e}-04$ & - & $2.1261 \mathrm{e}-04$ & - \\
& $1 / 16$ & $8.3929 \mathrm{e}-05$ & 1.9928 & $5.3418 \mathrm{e}-05$ & 1.9928 \\
& $1 / 32$ & $2.1014 \mathrm{e}-05$ & 1.9978 & $1.3374 \mathrm{e}-05$ & 1.9979 \\
& $1 / 64$ & $5.2426 \mathrm{e}-06$ & 2.0030 & $3.3366 \mathrm{e}-06$ & 2.0030 \\
& $1 / 128$ & $1.2947 \mathrm{e}-06$ & 2.0177 & $8.2404 \mathrm{e}-07$ & 2.0176 \\
\hline 0.90 & $1 / 8$ & $6.5352 \mathrm{e}-04$ & - & $4.1601 \mathrm{e}-04$ & - \\
& $1 / 16$ & $1.6365 \mathrm{e}-04$ & 1.9976 & $1.0417 \mathrm{e}-04$ & 1.9976 \\
& $1 / 32$ & $4.0921 \mathrm{e}-05$ & 1.9997 & $2.6048 \mathrm{e}-05$ & 1.9997 \\
$1 / 64$ & $1.0216 \mathrm{e}-05$ & 2.0020 & $6.5027 \mathrm{e}-06$ & 2.0021 \\
& $1 / 128$ & $2.5376 \mathrm{e}-06$ & 2.0093 & $1.6153 \mathrm{e}-06$ & 2.0093 \\
\hline 0.99 & $1 / 8$ & $7.1584 \mathrm{e}-04$ & - & $4.5567 \mathrm{e}-04$ & - \\
& $1 / 16$ & $1.7899 \mathrm{e}-04$ & 1.9998 & $1.1395 \mathrm{e}-04$ & 1.9996 \\
& $1 / 32$ & $4.4733 \mathrm{e}-05$ & 2.0004 & $2.8479 \mathrm{e}-05$ & 2.0005 \\
$1 / 64$ & $1.1167 \mathrm{e}-05$ & 2.0021 & $7.1095 \mathrm{e}-06$ & 2.0021 \\
& $1 / 128$ & $2.7756 \mathrm{e}-06$ & 2.0084 & $1.7671 \mathrm{e}-06$ & 2.0084 \\
\hline
\end{tabular}
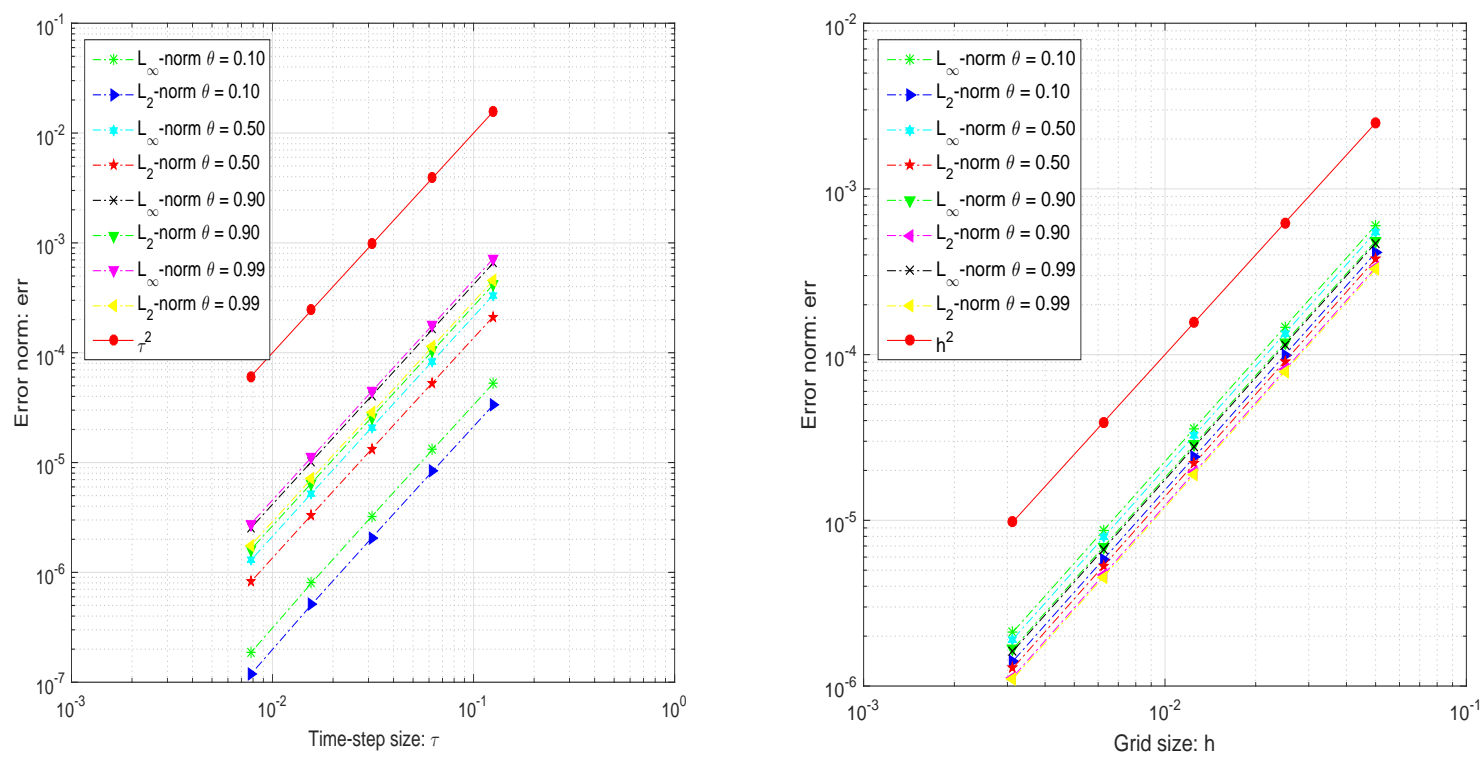

Fig. 1: Comparison the order of accuracy obtained by our proposed IDS for Example 1 in space and time variables. Left: time direction; Right: space direction. 
Table 2: $L_{2}$-norm and maximum norm error behavior versus grid size reduction when $\alpha=0.6$, $\beta=1.8$, and $\tau=h$ in Example 1 .

\begin{tabular}{cccccc}
\hline$\theta$ & $\tau$ & $\|\xi\|_{\mathcal{C}\left(\bar{\omega}_{h \tau}\right)}$ & CO in $\|\cdot\|_{\mathcal{C}\left(\bar{\omega}_{h \tau}\right)}$ & $\max _{0 \leq n \leq M}\left\|\xi^{n}\right\|_{0}$ & CO in $\|\cdot\|_{0}$ \\
\hline 0.10 & $1 / 20$ & $6.0326 \mathrm{e}-04$ & - & $4.1160 \mathrm{e}-04$ & - \\
& $1 / 40$ & $1.4719 \mathrm{e}-04$ & 2.0351 & $9.9593 \mathrm{e}-05$ & 2.0471 \\
& $1 / 80$ & $3.5781 \mathrm{e}-05$ & 2.0404 & $2.4068 \mathrm{e}-05$ & 2.0489 \\
& $1 / 160$ & $8.6941 \mathrm{e}-06$ & 2.0411 & $5.8187 \mathrm{e}-06$ & 2.0483 \\
$1 / 320$ & $2.1128 \mathrm{e}-06$ & 2.0409 & $1.4087 \mathrm{e}-06$ & 2.0464 \\
\hline 0.50 & $1 / 20$ & $5.5002 \mathrm{e}-04$ & - & $3.7743 \mathrm{e}-04$ & - \\
& $1 / 40$ & $1.3407 \mathrm{e}-04$ & 2.0365 & $9.1172 \mathrm{e}-05$ & 2.0496 \\
& $1 / 80$ & $3.2522 \mathrm{e}-05$ & 2.0435 & $2.1985 \mathrm{e}-05$ & 2.0520 \\
& $1 / 160$ & $7.8829 \mathrm{e}-06$ & 2.0446 & $5.3038 \mathrm{e}-06$ & 2.0514 \\
$1 / 320$ & $1.9110 \mathrm{e}-06$ & 2.0444 & $1.2815 \mathrm{e}-06$ & 2.0492 \\
\hline 0.90 & $1 / 20$ & $4.8566 \mathrm{e}-04$ & - & $3.3574 \mathrm{e}-04$ & - \\
& $1 / 40$ & $1.1823 \mathrm{e}-04$ & 2.0384 & $8.0954 \mathrm{e}-05$ & 2.0522 \\
$1 / 80$ & $2.8599 \mathrm{e}-05$ & 2.0475 & $1.9472 \mathrm{e}-05$ & 2.0557 \\
& $1 / 160$ & $6.9105 \mathrm{e}-06$ & 2.0491 & $4.6857 \mathrm{e}-06$ & 2.0551 \\
$1 / 320$ & $1.6699 \mathrm{e}-06$ & 2.0490 & $1.1296 \mathrm{e}-06$ & 2.0524 \\
\hline 0.99 & $1 / 20$ & $4.7183 \mathrm{e}-04$ & - & $3.2657 \mathrm{e}-04$ & - \\
& $1 / 40$ & $1.1479 \mathrm{e}-04$ & 2.0392 & $7.8721 \mathrm{e}-05$ & 2.0526 \\
& $1 / 80$ & $2.7752 \mathrm{e}-05$ & 2.0484 & $1.8925 \mathrm{e}-05$ & 2.0564 \\
$1 / 160$ & $6.7015 \mathrm{e}-06$ & 2.0500 & $4.5520 \mathrm{e}-06$ & 2.0558 \\
$1 / 320$ & $1.6181 \mathrm{e}-06$ & 2.0502 & $1.0969 \mathrm{e}-06$ & 2.0530 \\
\hline
\end{tabular}
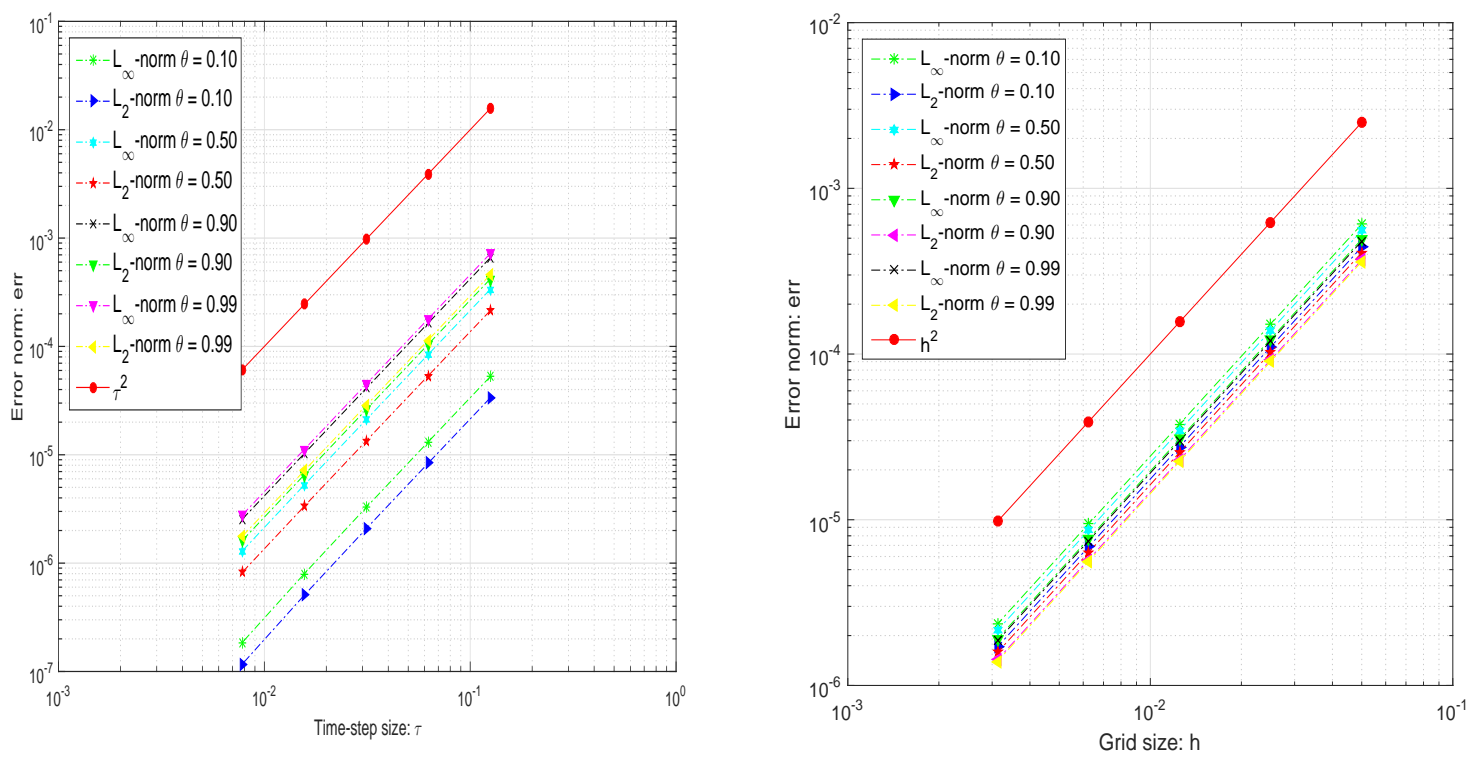

Fig. 2: Comparison the order of accuracy obtained by our proposed IDS for Example 1 in space and time variables. Left: time direction; Right: space direction.

Example 2. In this example, we consider the nonlinear equation on space interval $[0, L]=[0,1]$ 
Table 3: $L_{2}$-norm and maximum norm error behavior versus $\tau$-grid size reduction when $\alpha=0.99$, $\beta=1.99$, and $h=1 / 3000$ in Example 1 .

\begin{tabular}{cccccc}
\hline$\theta$ & $\tau$ & $\|\xi\|_{\mathcal{C}\left(\bar{\omega}_{h \tau}\right)}$ & CO in $\|\cdot\|_{\mathcal{C}\left(\bar{\omega}_{h \tau}\right)}$ & $\max _{0 \leq n \leq M}\left\|\xi^{n}\right\|_{0}$ & CO in $\|\cdot\|_{0}$ \\
\hline 0.10 & $1 / 8$ & $5.2548 \mathrm{e}-05$ & - & $3.3453 \mathrm{e}-05$ & - \\
& $1 / 16$ & $1.3149 \mathrm{e}-05$ & 1.9987 & $8.3697 \mathrm{e}-06$ & 1.9989 \\
& $1 / 32$ & $3.2701 \mathrm{e}-06$ & 2.0076 & $2.0799 \mathrm{e}-06$ & 2.0086 \\
& $1 / 64$ & $7.9770 \mathrm{e}-07$ & 2.0354 & $5.0584 \mathrm{e}-07$ & 2.0398 \\
& $1 / 128$ & $1.8525 \mathrm{e}-07$ & 2.1064 & $1.1708 \mathrm{e}-07$ & 2.1112 \\
\hline 0.50 & $1 / 8$ & $3.3536 \mathrm{e}-04$ & - & $2.1354 \mathrm{e}-04$ & - \\
& $1 / 16$ & $8.4257 \mathrm{e}-05$ & 1.9929 & $5.3650 \mathrm{e}-05$ & 1.9929 \\
& $1 / 32$ & $2.1094 \mathrm{e}-05$ & 1.9980 & $1.3430 \mathrm{e}-05$ & 1.9982 \\
$1 / 64$ & $5.2595 \mathrm{e}-06$ & 2.0038 & $3.3469 \mathrm{e}-06$ & 2.0045 \\
& $1 / 128$ & $1.2956 \mathrm{e}-06$ & 2.0213 & $8.2291 \mathrm{e}-07$ & 2.0240 \\
\hline 0.90 & $1 / 8$ & $6.5583 \mathrm{e}-04$ & - & $4.1765 \mathrm{e}-04$ & - \\
& $1 / 16$ & $1.6423 \mathrm{e}-04$ & 1.9976 & $1.0458 \mathrm{e}-04$ & 1.9976 \\
& $1 / 32$ & $4.1065 \mathrm{e}-05$ & 1.9997 & $2.6150 \mathrm{e}-05$ & 1.9998 \\
$1 / 64$ & $1.0249 \mathrm{e}-05$ & 2.0024 & $6.5248 \mathrm{e}-06$ & 2.0028 \\
& $1 / 128$ & $2.5427 \mathrm{e}-06$ & 2.0111 & $1.6172 \mathrm{e}-06$ & 2.0124 \\
\hline 0.99 & $1 / 8$ & $7.1823 \mathrm{e}-04$ & - & $4.5737 \mathrm{e}-04$ & - \\
& $1 / 16$ & $1.7958 \mathrm{e}-04$ & 1.9998 & $1.1437 \mathrm{e}-04$ & 1.9997 \\
& $1 / 32$ & $4.4877 \mathrm{e}-05$ & 2.0005 & $2.8580 \mathrm{e}-05$ & 2.0006 \\
$1 / 64$ & $1.1200 \mathrm{e}-05$ & 2.0025 & $7.1310 \mathrm{e}-06$ & 2.0028 \\
& $1 / 128$ & $2.7804 \mathrm{e}-06$ & 2.0101 & $1.7688 \mathrm{e}-06$ & 2.0113 \\
\hline
\end{tabular}

and time interval $[0, T]=[0,1]$ with advection coefficients $d_{+}(t)=\operatorname{sech}(t), d_{-}(t)=4 \operatorname{sech}(-t)$, diffusion coefficients $e_{+}(t)=(2+\cos (t))^{2}, e_{-}(t)=2+\cos ^{2}(t)$, and nonlinear term

$$
\begin{aligned}
g(u(x, t), x, t)= & \frac{\sin (u(x, t))}{4}+\left(\frac{\Gamma(3+\theta)}{\Gamma(3)} t^{2}+2 t^{1-\theta} E_{1,2-\theta}(2 t)\right) x^{2}(1-x)^{2} \\
& +\left(t^{2+\theta}+\exp (2 t)\right)\left\{\frac{\Gamma(3)}{\Gamma(3-\alpha)}\left[d_{+} x^{2-\alpha}+d_{-}(1-x)^{2-\alpha}\right]\right. \\
& \left.-\frac{2 \Gamma(4)}{\Gamma(4-\alpha)}\left[d_{+} x^{3-\alpha}+d_{-}(1-x)^{3-\alpha}\right]+\frac{\Gamma(5)}{\Gamma(5-\alpha)}\left[d_{+} x^{4-\alpha}+d_{-}(1-x)^{4-\alpha}\right]\right\} \\
& -t^{2+\theta}\left\{\frac{\Gamma(3)}{\Gamma(3-\beta)}\left[e_{+} x^{2-\beta}+e_{-}(1-x)^{2-\beta}\right]-\frac{2 \Gamma(4)}{\Gamma(4-\beta)}\left[e_{+} x^{3-\beta}+e_{-}(1-x)^{3-\beta}\right]\right. \\
& \left.+\frac{\Gamma(5)}{\Gamma(5-\beta)}\left[e_{+} x^{4-\beta}+e_{-}(1-x)^{4-\beta}\right]\right\}-\frac{\sin \left(\left(t^{2+\theta}+\exp (2 t)\right) x^{2}(1-x)^{2}\right)}{4},
\end{aligned}
$$

in which $E_{\mu, \nu}(z)$ is the Mittag-Leffler function with two parameters defined by

$$
E_{\mu, \nu}(z)=\sum_{k=0}^{\infty} \frac{z^{k}}{\Gamma(\mu k+\nu)} .
$$

The exact solution is $u(x, t)=\left(t^{2+\theta}+\exp (2 t)\right) x^{2}(1-x)^{2}$. For the finite difference discretization, the space step and time step are taken to be $h=1 / N$ and $\tau=h$, respectively. The errors 
Table 4: $L_{2}$-norm and maximum norm error behavior versus grid size reduction when $\alpha=0.99$, $\beta=1.99$, and $\tau=h$ in Example 1.

\begin{tabular}{cccccc}
\hline$\theta$ & $\tau$ & $\|\xi\|_{\mathcal{C}\left(\bar{\omega}_{h \tau}\right)}$ & CO in $\|\cdot\|_{\mathcal{C}\left(\bar{\omega}_{h \tau}\right)}$ & $\max _{0 \leq n \leq M}\left\|\xi^{n}\right\|_{0}$ & CO in $\|\cdot\|_{0}$ \\
\hline 0.10 & $1 / 20$ & $6.0735 \mathrm{e}-04$ & - & $4.4270 \mathrm{e}-04$ & - \\
& $1 / 40$ & $1.5155 \mathrm{e}-04$ & 2.0027 & $1.1041 \mathrm{e}-04$ & 2.0035 \\
& $1 / 80$ & $3.7819 \mathrm{e}-05$ & 2.0026 & $2.7528 \mathrm{e}-05$ & 2.0039 \\
& $1 / 160$ & $9.4336 \mathrm{e}-06$ & 2.0032 & $6.8625 \mathrm{e}-06$ & 2.0041 \\
& $1 / 320$ & $2.3531 \mathrm{e}-06$ & 2.0033 & $1.7107 \mathrm{e}-06$ & 2.0041 \\
\hline 0.50 & $1 / 20$ & $5.5527 \mathrm{e}-04$ & - & $4.0960 \mathrm{e}-04$ & - \\
& $1 / 40$ & $1.3860 \mathrm{e}-04$ & 2.0022 & $1.0219 \mathrm{e}-04$ & 2.0030 \\
& $1 / 80$ & $3.4589 \mathrm{e}-05$ & 2.0026 & $2.5475 \mathrm{e}-05$ & 2.0041 \\
& $1 / 160$ & $8.6263 \mathrm{e}-06$ & 2.0035 & $6.3494 \mathrm{e}-06$ & 2.0044 \\
& $1 / 320$ & $2.1512 \mathrm{e}-06$ & 2.0036 & $1.5825 \mathrm{e}-06$ & 2.0045 \\
\hline 0.90 & $1 / 20$ & $4.9287 \mathrm{e}-04$ & - & $3.6976 \mathrm{e}-04$ & - \\
& $1 / 40$ & $1.2317 \mathrm{e}-04$ & 2.0006 & $9.2308 \mathrm{e}-05$ & 2.0021 \\
& $1 / 80$ & $3.0734 \mathrm{e}-05$ & 2.0027 & $2.3012 \mathrm{e}-05$ & 2.0041 \\
& $1 / 160$ & $7.6637 \mathrm{e}-06$ & 2.0037 & $5.7346 \mathrm{e}-06$ & 2.0046 \\
& $1 / 320$ & $1.9108 \mathrm{e}-06$ & 2.0039 & $1.4289 \mathrm{e}-06$ & 2.0048 \\
\hline 0.99 & $1 / 20$ & $4.7956 \mathrm{e}-04$ & - & $3.6117 \mathrm{e}-04$ & - \\
& $1 / 40$ & $1.1989 \mathrm{e}-04$ & 2.0000 & $9.0184 \mathrm{e}-05$ & 2.0017 \\
& $1 / 80$ & $2.9914 \mathrm{e}-05$ & 2.0028 & $2.2483 \mathrm{e}-05$ & 2.0041 \\
& $1 / 160$ & $7.4589 \mathrm{e}-06$ & 2.0038 & $5.6023 \mathrm{e}-06$ & 2.0047 \\
& $1 / 320$ & $1.8596 \mathrm{e}-06$ & 2.0040 & $1.3958 \mathrm{e}-06$ & 2.0049 \\
\hline
\end{tabular}

$(\xi=U-u)$ and convergence order $(\mathrm{CO})$ in the norms $\|\cdot\|_{0}$ and $\|\cdot\|_{\mathcal{C}\left(\bar{\omega}_{h \tau}\right)}$, where $\|U\|_{\mathcal{C}\left(\bar{\omega}_{h \tau}\right)}=$ $\max _{\left(x_{i}, t_{j}\right) \in \bar{\omega}_{h \tau}}|U|$, are given in Example 1 .

As can be seen in Tables 5, the numerical solution provided by the difference approximation is in good agreement with our theoretical analysis. In Tables 5 and 7 , we take $h=1 / 800$, the errors in maximum norm and $L_{2}$-norm decrease steadily with the shortening of time step, and the convergence order of time is the expected $\mathcal{O}\left(\tau^{2}\right)$. While in Tables 6 and 8 , the mesh size $\tau=h$ is chosen and the spatial convergence rates of the scheme (2.10) are also nearly two, for $\theta=0.1,0.5,0.9,0.99$. Furthermore, Figs. 3 and 4 are plotted to further illustrate the reliability of our proposed scheme, i.e., the slopes of the error curves in this log-log plot are 2, for $\theta=0.1,0.5,0.9,0.99$. 
Table 5: $L_{2}$-norm and maximum norm error behavior versus $\tau$-grid size reduction when $\alpha=0.6$, $\beta=1.8$ and $h=1 / 800$ in Example 2 .

\begin{tabular}{cccccc}
\hline$\theta$ & $\tau$ & $\|\xi\|_{\mathcal{C}\left(\bar{\omega}_{h \tau}\right)}$ & CO in $\|\cdot\|_{\mathcal{C}\left(\bar{\omega}_{h \tau}\right)}$ & $\max _{0 \leq n \leq M}\left\|\xi^{n}\right\|_{0}$ & CO in $\|\cdot\|_{0}$ \\
\hline 0.10 & $1 / 8$ & $6.7718 \mathrm{e}-04$ & - & $4.3108 \mathrm{e}-04$ & - \\
& $1 / 16$ & $1.7300 \mathrm{e}-04$ & 1.9688 & $1.1009 \mathrm{e}-04$ & 1.9693 \\
& $1 / 32$ & $4.1798 \mathrm{e}-05$ & 2.0493 & $2.6564 \mathrm{e}-05$ & 2.0511 \\
& $1 / 64$ & $8.3378 \mathrm{e}-06$ & 2.3257 & $5.2789 \mathrm{e}-06$ & 2.3312 \\
& $1 / 128$ & $1.6425 \mathrm{e}-06$ & 2.3438 & $4.9654 \mathrm{e}-07$ & 3.4103 \\
\hline 0.50 & $1 / 8$ & $2.8747 \mathrm{e}-03$ & - & $1.8297 \mathrm{e}-03$ & - \\
& $1 / 16$ & $7.3181 \mathrm{e}-04$ & 1.9739 & $4.6572 \mathrm{e}-04$ & 1.9741 \\
& $1 / 32$ & $1.8254 \mathrm{e}-04$ & 2.0033 & $1.1613 \mathrm{e}-04$ & 2.0037 \\
& $1 / 64$ & $4.3648 \mathrm{e}-05$ & 2.0642 & $2.7733 \mathrm{e}-05$ & 2.0661 \\
& $1 / 128$ & $8.7404 \mathrm{e}-06$ & 2.3201 & $5.5326 \mathrm{e}-06$ & 2.3256 \\
\hline 0.90 & $1 / 8$ & $4.0951 \mathrm{e}-03$ & - & $2.6098 \mathrm{e}-03$ & - \\
& $1 / 16$ & $1.0327 \mathrm{e}-03$ & 1.9875 & $6.5724 \mathrm{e}-04$ & 1.9894 \\
& $1 / 32$ & $2.5667 \mathrm{e}-04$ & 2.0084 & $1.6330 \mathrm{e}-04$ & 2.0089 \\
& $1 / 64$ & $6.2038 \mathrm{e}-05$ & 2.0487 & $3.9436 \mathrm{e}-05$ & 2.0499 \\
& $1 / 128$ & $1.3330 \mathrm{e}-05$ & 2.2185 & $8.4480 \mathrm{e}-06$ & 2.2228 \\
\hline 0.99 & $1 / 8$ & $4.1885 \mathrm{e}-03$ & - & $2.6801 \mathrm{e}-03$ & - \\
& $1 / 16$ & $1.0594 \mathrm{e}-03$ & 1.9832 & $6.7470 \mathrm{e}-04$ & 1.9900 \\
& $1 / 32$ & $2.6328 \mathrm{e}-04$ & 2.0086 & $1.6752 \mathrm{e}-04$ & 2.0099 \\
$1 / 64$ & $6.3658 \mathrm{e}-05$ & 2.0482 & $4.0472 \mathrm{e}-05$ & 2.0493 \\
& $1 / 128$ & $1.3737 \mathrm{e}-05$ & 2.2123 & $8.7084 \mathrm{e}-06$ & 2.2164 \\
\hline
\end{tabular}
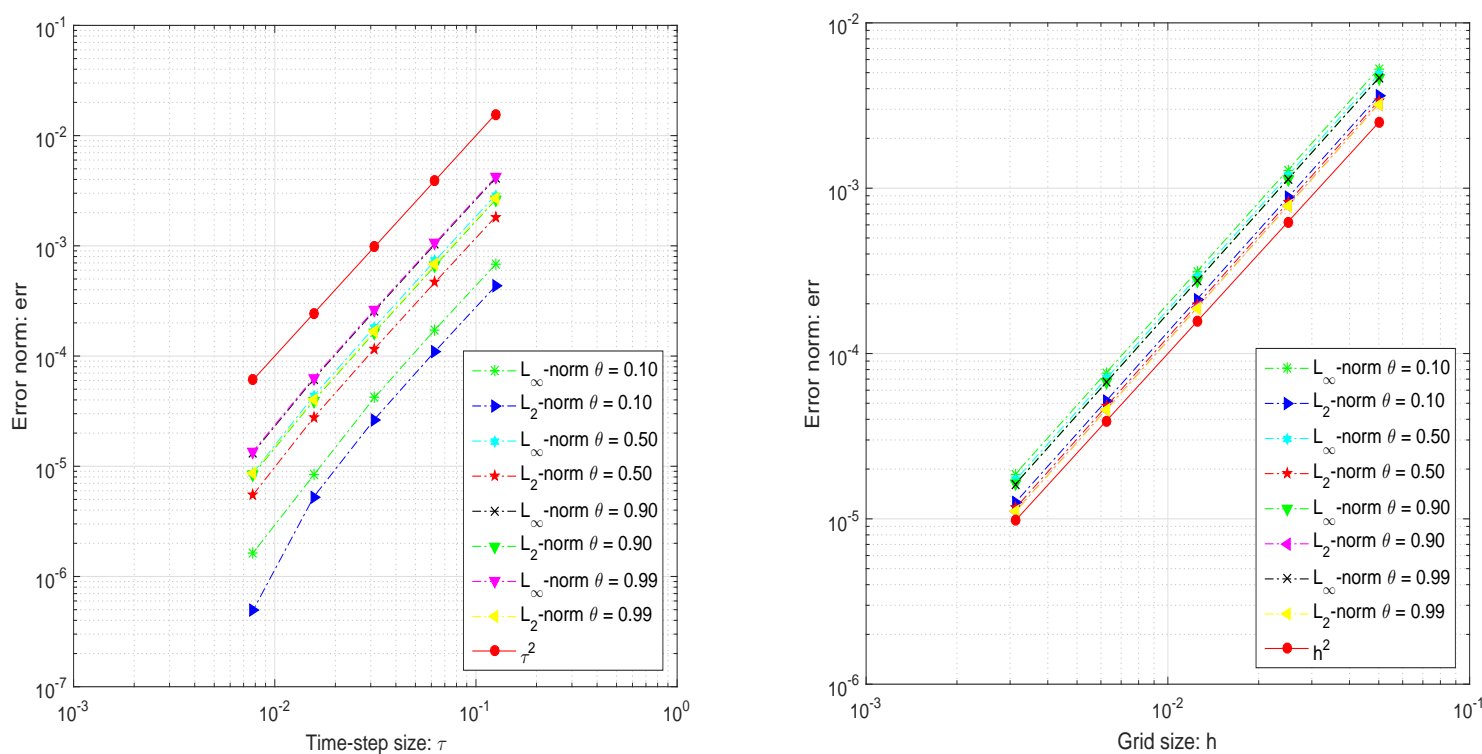

Fig. 3: Comparison the order of accuracy obtained by our proposed IDS for Example 2 in space and time variables. Left: time direction; Right: space direction. 
Table 6: $L_{2}$-norm and maximum norm error behavior versus grid size reduction when $\alpha=0.6$, $\beta=1.8$ and $\tau=h$ in Example 2 .

\begin{tabular}{cccccc}
\hline$\theta$ & $\tau$ & $\|\xi\|_{\mathcal{C}\left(\bar{\omega}_{h \tau}\right)}$ & CO in $\|\cdot\|_{\mathcal{C}\left(\bar{\omega}_{h \tau}\right)}$ & $\max _{0 \leq n \leq M}\left\|\xi^{n}\right\|_{0}$ & CO in $\|\cdot\|_{0}$ \\
\hline 0.10 & $1 / 20$ & $5.2772 \mathrm{e}-03$ & - & $3.6233 \mathrm{e}-03$ & - \\
& $1 / 40$ & $1.2913 \mathrm{e}-03$ & 2.0309 & $8.7967 \mathrm{e}-04$ & 2.0423 \\
& $1 / 80$ & $3.1470 \mathrm{e}-04$ & 2.0368 & $2.1331 \mathrm{e}-04$ & 2.0440 \\
& $1 / 160$ & $7.6674 \mathrm{e}-05$ & 2.0372 & $5.1742 \mathrm{e}-05$ & 2.0435 \\
& $1 / 320$ & $1.8683 \mathrm{e}-05$ & 2.0370 & $1.2566 \mathrm{e}-05$ & 2.0418 \\
\hline 0.50 & $1 / 20$ & $4.8930 \mathrm{e}-03$ & - & $3.3744 \mathrm{e}-03$ & - \\
& $1 / 40$ & $1.1943 \mathrm{e}-03$ & 2.0346 & $8.1793 \mathrm{e}-04$ & 2.0446 \\
& $1 / 80$ & $2.9056 \mathrm{e}-04$ & 2.0393 & $1.9797 \mathrm{e}-04$ & 2.0467 \\
& $1 / 160$ & $7.0647 \mathrm{e}-05$ & 2.0401 & $4.7934 \mathrm{e}-05$ & 2.0462 \\
& $1 / 320$ & $1.7181 \mathrm{e}-05$ & 2.0398 & $1.1621 \mathrm{e}-05$ & 2.0443 \\
\hline 0.90 & $1 / 20$ & $4.6652 \mathrm{e}-03$ & - & $3.2255 \mathrm{e}-03$ & - \\
& $1 / 40$ & $1.1378 \mathrm{e}-03$ & 2.0357 & $7.8171 \mathrm{e}-04$ & 2.0448 \\
& $1 / 80$ & $2.7670 \mathrm{e}-04$ & 2.0399 & $1.8908 \mathrm{e}-04$ & 2.0476 \\
& $1 / 160$ & $6.7216 \mathrm{e}-05$ & 2.0414 & $4.5748 \mathrm{e}-05$ & 2.0472 \\
& $1 / 320$ & $1.6330 \mathrm{e}-05$ & 2.0413 & $1.1083 \mathrm{e}-05$ & 2.0454 \\
\hline 0.99 & $1 / 20$ & $4.6390 \mathrm{e}-03$ & - & $3.2055 \mathrm{e}-03$ & - \\
& $1 / 40$ & $1.1310 \mathrm{e}-03$ & 2.0362 & $7.7718 \mathrm{e}-04$ & 2.0442 \\
& $1 / 80$ & $2.7508 \mathrm{e}-04$ & 2.0397 & $1.8801 \mathrm{e}-04$ & 2.0474 \\
& $1 / 160$ & $6.6816 \mathrm{e}-05$ & 2.0416 & $4.5488 \mathrm{e}-05$ & 2.0473 \\
$1 / 320$ & $1.6231 \mathrm{e}-05$ & 2.0414 & $1.1020 \mathrm{e}-05$ & 2.0454 \\
\hline
\end{tabular}
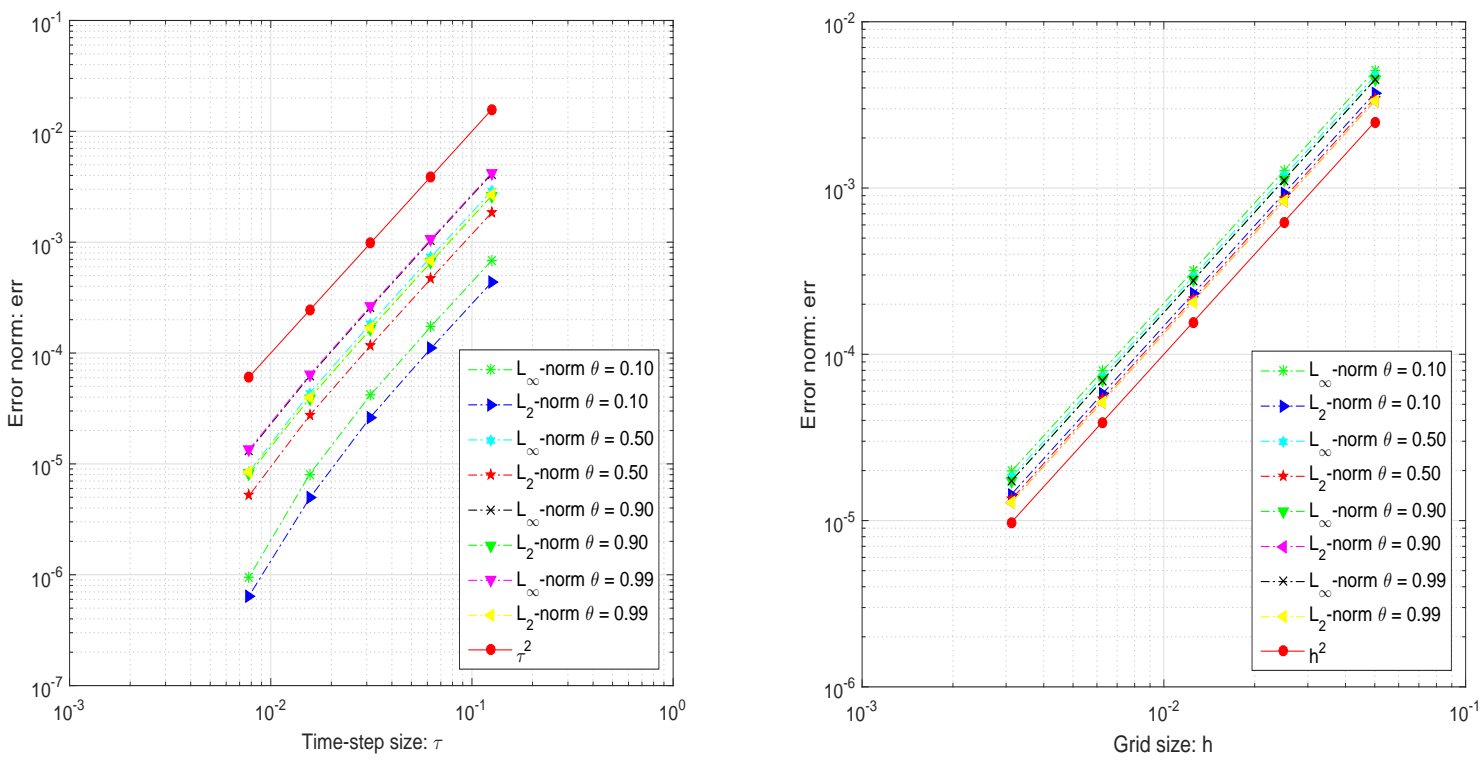

Fig. 4: Comparison the order of accuracy obtained by our proposed IDS for Example 2 in space and time variables. Left: time direction; Right: space direction. 
Table 7: $L_{2}$-norm and maximum norm error behavior versus $\tau$-grid size reduction when $\alpha=0.99$, $\beta=1.99$ and $h=1 / 800$ in Example 2 .

\begin{tabular}{cccccc}
\hline$\theta$ & $\tau$ & $\|\xi\|_{\mathcal{C}\left(\bar{\omega}_{h \tau}\right)}$ & CO in $\|\cdot\|_{\mathcal{C}\left(\bar{\omega}_{h \tau}\right)}$ & $\max _{0 \leq n \leq M}\left\|\xi^{n}\right\|_{0}$ & CO in $\|\cdot\|_{0}$ \\
\hline 0.10 & $1 / 8$ & $6.7919 \mathrm{e}-04$ & - & $4.3233 \mathrm{e}-04$ & - \\
& $1 / 16$ & $1.7333 \mathrm{e}-04$ & 1.9703 & $1.1015 \mathrm{e}-04$ & 1.9727 \\
& $1 / 32$ & $4.1678 \mathrm{e}-05$ & 2.0562 & $2.6300 \mathrm{e}-05$ & 2.0663 \\
& $1 / 64$ & $8.0974 \mathrm{e}-06$ & 2.3638 & $4.9278 \mathrm{e}-06$ & 2.4160 \\
& $1 / 128$ & $9.5365 \mathrm{e}-07$ & 3.0859 & $6.4747 \mathrm{e}-07$ & 2.9281 \\
\hline 0.50 & $1 / 8$ & $2.8857 \mathrm{e}-03$ & - & $1.8374 \mathrm{e}-03$ & - \\
& $1 / 16$ & $7.3455 \mathrm{e}-04$ & 1.9740 & $4.6750 \mathrm{e}-04$ & 1.9746 \\
& $1 / 32$ & $1.8305 \mathrm{e}-04$ & 2.0046 & $1.1631 \mathrm{e}-04$ & 2.0070 \\
$1 / 64$ & $4.3571 \mathrm{e}-05$ & 2.0708 & $2.7500 \mathrm{e}-05$ & 2.0805 \\
& $1 / 128$ & $8.5104 \mathrm{e}-06$ & 2.3561 & $5.1893 \mathrm{e}-06$ & 2.4058 \\
\hline 0.90 & $1 / 8$ & $4.1053 \mathrm{e}-03$ & - & $2.6172 \mathrm{e}-03$ & - \\
& $1 / 16$ & $1.0367 \mathrm{e}-03$ & 1.9855 & $6.5998 \mathrm{e}-04$ & 1.9875 \\
& $1 / 32$ & $2.5752 \mathrm{e}-04$ & 2.0092 & $1.6373 \mathrm{e}-04$ & 2.0111 \\
$1 / 64$ & $6.2046 \mathrm{e}-05$ & 2.0533 & $3.9263 \mathrm{e}-05$ & 2.0601 \\
& $1 / 128$ & $1.3122 \mathrm{e}-05$ & 2.2414 & $8.1204 \mathrm{e}-06$ & 2.2735 \\
\hline 0.99 & $1 / 8$ & $4.1843 \mathrm{e}-03$ & - & $2.6789 \mathrm{e}-03$ & - \\
& $1 / 16$ & $1.0619 \mathrm{e}-03$ & 1.9783 & $6.7724 \mathrm{e}-04$ & 1.9839 \\
& $1 / 32$ & $2.6411 \mathrm{e}-04$ & 2.0074 & $1.6791 \mathrm{e}-04$ & 2.0120 \\
$1 / 64$ & $6.3651 \mathrm{e}-05$ & 2.0529 & $4.0288 \mathrm{e}-05$ & 2.0593 \\
& $1 / 128$ & $1.3524 \mathrm{e}-05$ & 2.2347 & $8.3771 \mathrm{e}-06$ & 2.2658 \\
\hline
\end{tabular}

\subsection{Fast implementations}

Example 3. In this example, we consider the case of linear situation (1.1), and the exact solution is given in Example 1. In MATLAB code, we take the commands of generating matrices as the start point of Time1, and in the rest tables, "Speed-up" denotes $\frac{\text { Time1 }}{\text { Timek }}(k=2,3)$ and "Iter" is the average number of iterations required for solving the TSADE problem, namely,

$$
\text { Iter }=\frac{1}{M} \sum_{m=1}^{M} \operatorname{Iter}(m),
$$

where Iter $(m)$ represents the number of iterations required for solving (3.1). As for $\operatorname{GPBiCOR}(m$, $\ell)$ method [42] with circulant preconditioners in this example, a large number of experiments reveal that the results are better than others, when $m=3$ and $\ell=1$. Some eigenvalue plots about both original and preconditioned matrices are drawn in Figs. [5]6. These two figures confirm that for circulant preconditioning, the eigenvalues of preconditioned matrices are clustered at 1 except for a few of them. That is to say, the vast majority of the eigenvalues are well separated away from 0 . We validate the effectiveness and robustness of the designed circulant preconditioner from the respective of clustering spectrum distribution.

The numerical results in Example 3 are presented in Tables 9, 10, reflecting that the average iterations of the two Krylov subspace methods with circulant preconditioners have little 
Table 8: $L_{2}$-norm and maximum norm error behavior versus grid size reduction when $\alpha=0.99$, $\beta=1.99$ and $\tau=h$ in Example 2.

\begin{tabular}{cccccc}
\hline$\theta$ & $\tau$ & $\|\xi\|_{\mathcal{C}\left(\bar{\omega}_{h \tau}\right)}$ & CO in $\|\cdot\|_{\mathcal{C}\left(\bar{\omega}_{h \tau}\right)}$ & $\max _{0 \leq n \leq M}\left\|\xi^{n}\right\|_{0}$ & CO in $\|\cdot\|_{0}$ \\
\hline 0.10 & $1 / 20$ & $5.1023 \mathrm{e}-03$ & - & $3.7233 \mathrm{e}-03$ & - \\
& $1 / 40$ & $1.2730 \mathrm{e}-03$ & 2.0029 & $9.2852 \mathrm{e}-04$ & 2.0036 \\
& $1 / 80$ & $3.1774 \mathrm{e}-04$ & 2.0023 & $2.3151 \mathrm{e}-04$ & 2.0039 \\
& $1 / 160$ & $7.9261 \mathrm{e}-05$ & 2.0032 & $5.7718 \mathrm{e}-05$ & 2.0040 \\
& $1 / 320$ & $1.9772 \mathrm{e}-05$ & 2.0032 & $1.4389 \mathrm{e}-05$ & 2.0041 \\
\hline 0.50 & $1 / 20$ & $4.7154 \mathrm{e}-03$ & - & $3.4794 \mathrm{e}-03$ & - \\
& $1 / 40$ & $1.1767 \mathrm{e}-03$ & 2.0026 & $8.6769 \mathrm{e}-04$ & 2.0036 \\
& $1 / 80$ & $2.9361 \mathrm{e}-04$ & 2.0028 & $2.1629 \mathrm{e}-04$ & 2.0042 \\
& $1 / 160$ & $7.3226 \mathrm{e}-05$ & 2.0035 & $5.3912 \mathrm{e}-05$ & 2.0043 \\
& $1 / 320$ & $1.8263 \mathrm{e}-05$ & 2.0034 & $1.3438 \mathrm{e}-05$ & 2.0043 \\
\hline 0.90 & $1 / 20$ & $4.4905 \mathrm{e}-03$ & - & $3.3363 \mathrm{e}-03$ & - \\
& $1 / 40$ & $1.1217 \mathrm{e}-03$ & 2.0012 & $8.3257 \mathrm{e}-04$ & 2.0026 \\
& $1 / 80$ & $2.7992 \mathrm{e}-04$ & 2.0026 & $2.0758 \mathrm{e}-04$ & 2.0039 \\
& $1 / 160$ & $6.9818 \mathrm{e}-05$ & 2.0033 & $5.1743 \mathrm{e}-05$ & 2.0042 \\
& $1 / 320$ & $1.7413 \mathrm{e}-05$ & 2.0034 & $1.2897 \mathrm{e}-05$ & 2.0043 \\
\hline 0.99 & $1 / 20$ & $4.4668 \mathrm{e}-03$ & - & $3.3193 \mathrm{e}-03$ & - \\
& $1 / 40$ & $1.1155 \mathrm{e}-03$ & 2.0016 & $8.2848 \mathrm{e}-04$ & 2.0023 \\
& $1 / 80$ & $2.7838 \mathrm{e}-04$ & 2.0026 & $2.0657 \mathrm{e}-04$ & 2.0038 \\
& $1 / 160$ & $6.9435 \mathrm{e}-05$ & 2.0033 & $5.1492 \mathrm{e}-05$ & 2.0042 \\
& $1 / 320$ & $1.7317 \mathrm{e}-05$ & 2.0035 & $1.2834 \mathrm{e}-05$ & 2.0044 \\
\hline
\end{tabular}

difference. In other words, the number of average iterations of these methods is about 6.6. On the other hand, the CPU time by the two Krylov subspace methods with circulant preconditioners are much less than that by LU factorization method as $M$ and $N$ become large. When $M=N=2^{10}$ in Table 9, the CPU time is about 18.5 seconds, and the Speed-up is almost 7.4, especially for PBiCGSTAB method, the Speed-up can reach about 8.21. At the same case, in Table 10 the CPU time is about 18.7 seconds, and the Speed-up is almost 7.7. Meanwhile, although Time1 required by LU factorization method for small test problems $\left(M=N=2^{6}, 2^{7}\right)$ is the cheapest among other two methods (i.e. PBiCGSTAB and $\operatorname{PGPBiCOR}(m, \ell)$ ), our proposed methods are still more attractive in aspects of the lower memory requirement.

Table 9: CPU time in seconds for solving Example 1 with $\theta=0.9, \alpha=0.8, \beta=1.9$.

\begin{tabular}{ccccccc}
\hline & & \multicolumn{2}{c}{ PBiCGSTAB } & & \multicolumn{2}{c}{ PGPBiCOR(3,1) } \\
\cline { 3 - 4 }$h=\tau$ & Time1 & Time2(Iter) & Speed-up & & Time3(Iter) & Speed-up \\
\hline $2^{-6}$ & 0.031 & $0.161(6.9)$ & 0.19 & & $0.213(5.6)$ & 0.14 \\
$2^{-7}$ & 0.145 & $0.488(7.0)$ & 0.30 & & $0.612(6.1)$ & 0.24 \\
$2^{-8}$ & 1.624 & $1.217(7.0)$ & 1.33 & & $1.500(6.3)$ & 1.08 \\
$2^{-9}$ & 17.375 & $4.828(7.0)$ & 3.60 & & $5.592(6.5)$ & 3.11 \\
$2^{-10}$ & 143.968 & $17.544(7.0)$ & 8.21 & & $19.393(6.7)$ & 7.42 \\
\hline
\end{tabular}



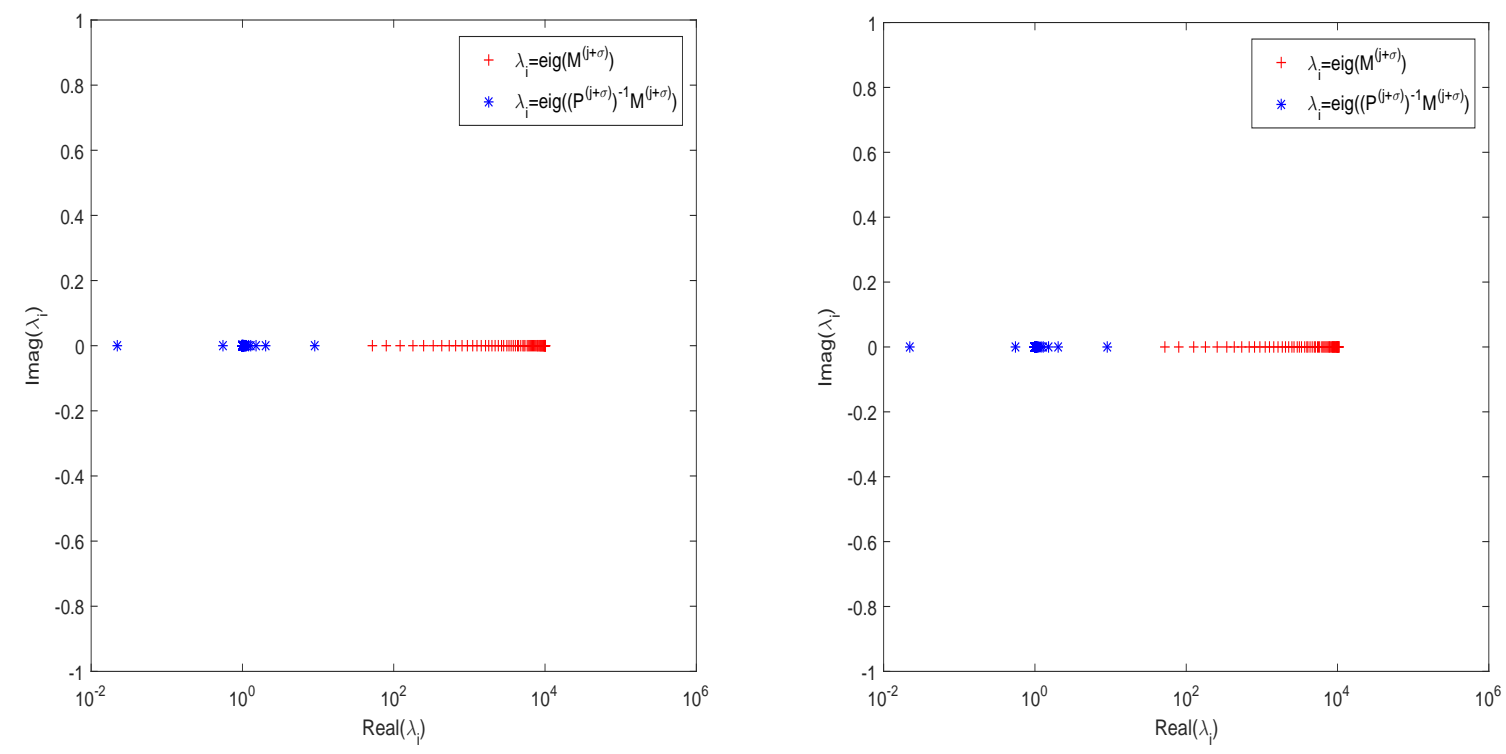

Fig. 5: Spectrum of both original and preconditioned matrices, when $M=N=2^{6}, \theta=0.9$, $\alpha=0.8, \beta=1.9$. Left: Time level $j=0$; Right: Time level $j=1$.

Table 10: CPU time in seconds for solving Example 1 with $\theta=0.7, \alpha=0.6, \beta=1.5$.

\begin{tabular}{ccccccc}
\hline & & \multicolumn{2}{c}{ PBiCGSTAB } & & \multicolumn{2}{c}{ PGPBiCOR(3,1) } \\
\cline { 3 - 4 } \cline { 6 - 7 }$h=\tau$ & Time1 & Time2(Iter) & Speed-up & & Time3(Iter) & Speed-up \\
\hline $2^{-6}$ & 0.031 & $0.167(6.8)$ & 0.18 & & $0.154(6.0)$ & 0.20 \\
$2^{-7}$ & 0.149 & $0.509(7.0)$ & 0.29 & & $0.485(6.3)$ & 0.31 \\
$2^{-8}$ & 1.701 & $1.335(7.8)$ & 1.27 & & $1.281(7.2)$ & 1.33 \\
$2^{-9}$ & 17.251 & $5.325(8.0)$ & 3.24 & & $5.275(7.6)$ & 3.27 \\
$2^{-10}$ & 143.739 & $19.166(8.1)$ & 7.50 & & $18.261(7.8)$ & 7.87 \\
\hline
\end{tabular}



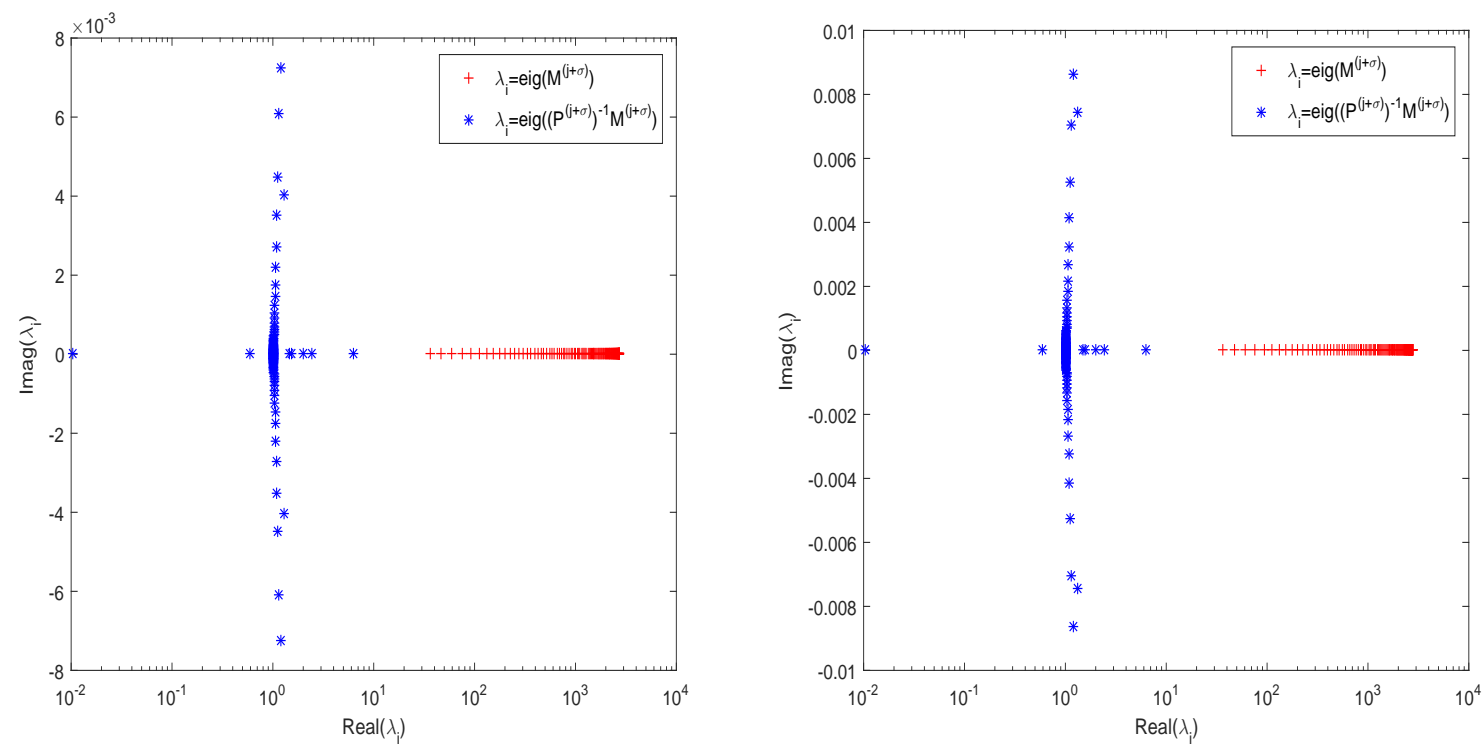

Fig. 6: Spectrum of both original and preconditioned matrices, when $M=N=2^{7}, \theta=0.7$, $\alpha=0.6, \beta=1.5$. Left: Time level $j=0$; Right: Time level $j=1$.

Example 4. In this example, the exact solution and nonlinear source term are given in Example 2. For performing algorithm (3.4)-(3.6), the outer iteration tolerance is chosen as $10^{-12}$. As for $\operatorname{GPBiCOR}(m, \ell)$ method [42] with circulant preconditioners in this example, a large number of experiments reveal that the results are better than others, when $m=3$ and $\ell=1$. Some eigenvalue plots about both original and preconditioned matrices are drawn in Figs. 7,8, These two figures confirm that for circulant preconditioning, the eigenvalues of preconditioned matrices are clustered at 1 except for a few of them. That is to say, the vast majority of the eigenvalues are well separated away from 0 . We validate the effectiveness and robustness of the designed circulant preconditioner from the respective of clustering spectrum distribution.

In Tables 11-12, "Iter1" represents the average number of iterations required for solving the nonlinear problem with algorithm (3.4)-(3.6), i.e.,

$$
\operatorname{Iter} 1=\frac{1}{M} \sum_{m=1}^{M} \operatorname{Iter} 1(m),
$$

in which Iter $1(m)$ is the number of outer iteration required to solve (3.4), and "Iter2" denotes the average iterative number of a preconditioned Krylov subspace method required to solve (3.4), namely,

$$
\text { Iter2 }=\frac{1}{M} \sum_{m=1}^{M} \frac{1}{\operatorname{Iter} 1(m)} \sum_{k=1}^{\operatorname{Iter} 1(m)} \operatorname{Iter} 2(k)
$$

in which Iter2 $(k)$ is the iterative number of the preconditioned Krylov subspace method required at $k$ th outer iteration. In view of the overall, the CPU time by the two Krylov subspace methods with circulant preconditioners are much less than that by LU factorization method as $M$ and $N$ become large. When $M=N=2^{10}$ in Table 11, the CPU time is about 41.8 seconds, and the Speed-up is almost 3.8. At the same case, Table 12 indicates that the CPU time is about 
52.3 seconds, and the Speed-up is almost 3.2. Meanwhile, although Time1 required by LU factorization method for small test problems $\left(M=N=2^{6}, 2^{7}, 2^{8}\right)$ is the cheapest among other two methods (i.e. PBiCGSTAB and $\operatorname{PGPBiCOR}(m, \ell)$ ), our proposed methods are still more attractive in aspects of the lower memory requirement. As a conclusion, our proposed IDS with fast solution techniques is still more practical than LU factorization method.

Table 11: CPU time in seconds for solving Example 2 with $\theta=0.9, \alpha=0.8, \beta=1.9$.

\begin{tabular}{ccccccc}
\hline & & \multicolumn{2}{c}{ PBiCGSTAB } & & \multicolumn{2}{c}{ PGPBiCOR $(3,1)$} \\
\cline { 3 - 4 } \cline { 6 - 7 }$h=\tau$ & Time1(Iter1) & Time2(Iter1, Iter2) & Speed-up & & Time3(Iter1, Iter2) & Speed-up \\
\hline $2^{-6}$ & $0.038(5.0)$ & $0.470(5.0,7.0)$ & 0.08 & & $0.482(5.0,6.0)$ & 0.08 \\
$2^{-7}$ & $0.164(4.1)$ & $1.414(4.1,7.0)$ & 0.12 & & $1.420(4.1,6.0)$ & 0.12 \\
$2^{-8}$ & $1.922(4.0)$ & $3.325(4.0,7.0)$ & 0.58 & & $3.350(4.0,6.0)$ & 0.57 \\
$2^{-9}$ & $19.122(4.0)$ & $13.923(4.0,7.0)$ & 1.37 & & $13.879(4.0,6.0)$ & 1.38 \\
$2^{-10}$ & $159.561(3.5)$ & $42.497(3.5,7.4)$ & 3.75 & & $41.005(3.5,6.0)$ & 3.89 \\
\hline
\end{tabular}
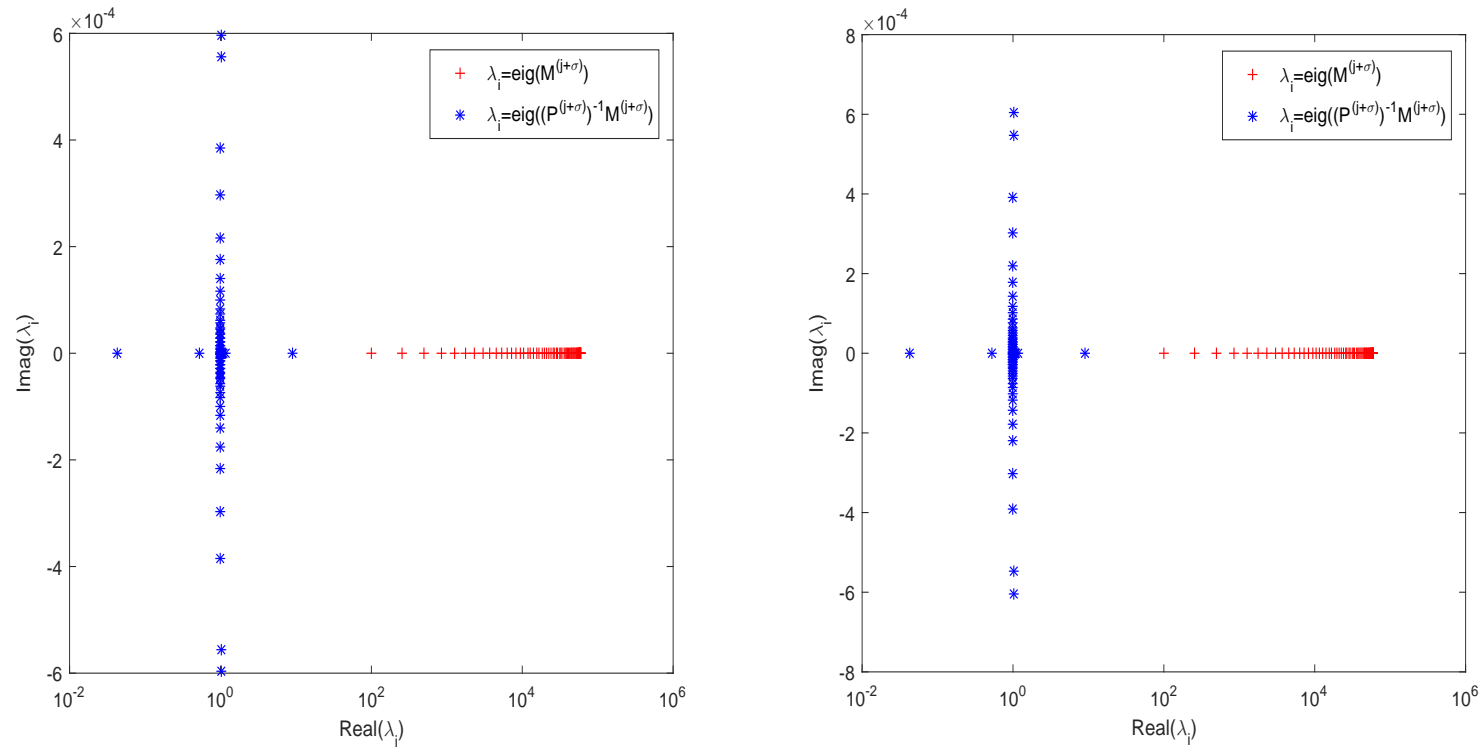

Fig. 7: Spectrum of both original and preconditioned matrices, when $M=N=2^{6}, \theta=0.9$, $\alpha=0.8, \beta=1.9$. Left: Time level $j=0$; Right: Time level $j=1$. 
Table 12: CPU time in seconds for solving Example 2 with $\theta=0.7, \alpha=0.6, \beta=1.7$.

\begin{tabular}{ccccccc}
\hline & & \multicolumn{2}{c}{ PBiCGSTAB } & & \multicolumn{2}{c}{ PGPBiCOR(3,1) } \\
\cline { 3 - 4 }$h=\tau$ & Time1(Iter1) & Time2(Iter1, Iter2) & Speed-up & & Time3(Iter1, Iter2) & Speed-up \\
\hline $2^{-6}$ & $0.037(5.0)$ & $0.480(5.0,7.0)$ & 0.08 & & $0.476(5.0,6.0)$ & 0.08 \\
$2^{-7}$ & $0.171(5.0)$ & $1.666(5.0,7.0)$ & 0.10 & & $1.866(5.0,7.0)$ & 0.09 \\
$2^{-8}$ & $1.802(4.3)$ & $3.975(4.3,8.1)$ & 0.45 & & $4.022(4.3,7.0)$ & 0.45 \\
$2^{-9}$ & $18.950(4.0)$ & $15.604(4.0,8.0)$ & 1.21 & & $15.873(4.0,7.2)$ & 1.19 \\
$2^{-10}$ & $164.387(4.0)$ & $50.313(4.0,8.0)$ & 3.27 & & $54.279(4.0,7.8)$ & 3.03 \\
\hline
\end{tabular}
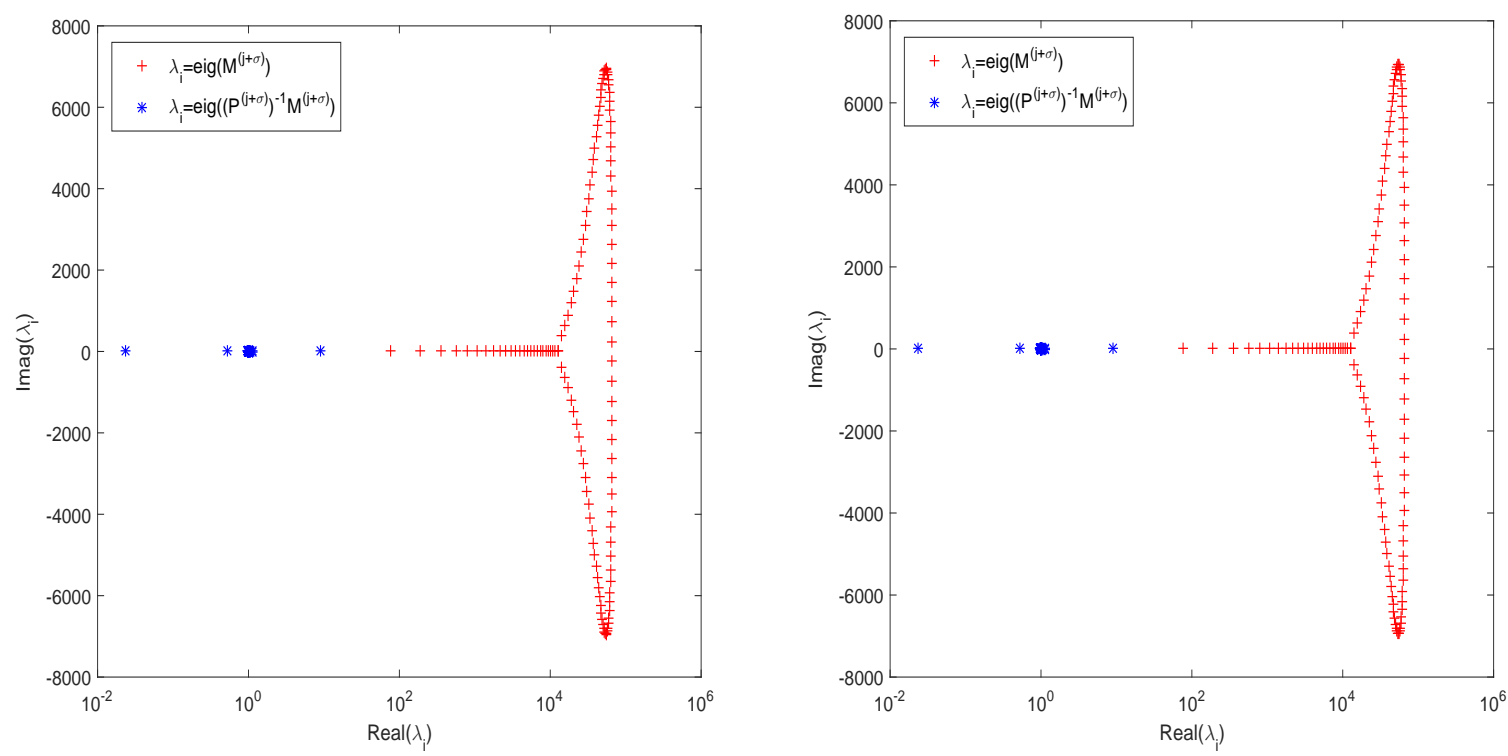

Fig. 8: Spectrum of both original and preconditioned matrices, when $M=N=2^{7}, \theta=0.7$, $\alpha=0.6, \beta=1.7$. Left: Time level $j=0$; Right: Time level $j=1$.

\section{Conclusion}

In this work, we constructed a difference scheme of the second order approximation in space and time for the linear TSADE with variable coefficients. Then, we utilized the same numerical approximation technique to simulate the nonlinear problem of TSADE and the convergence order still was $\mathcal{O}\left(\tau^{2}+h^{2}\right)$. Numerical tests shown that our implicit difference schemes converge with second-order accuracy in both time and space. More significantly, we employed two reliable preconditioning iterative techniques, which have only computational cost and memory of $\mathcal{O}(N \log N)$ and $\mathcal{O}(N)$ respectively, to ameliorate the calculation skill. And numerical experiments strongly support the efficiency of the two preconditioning methods. In our future work, we will apply our IDM into more linear/nonlinear fractional partial differential equations and make a rigorous theoretical analysis of the stability for the developed difference schemes. 


\section{Acknowledgments}

This work is supported by 973 Program (2013CB329404), NSFC (1370147, 11501085 and 61402082), the Fundamental Research Funds for the Central Universities (ZYGX2016J132 and ZYGX2016J138), the Scientific Research Fund of Sichuan Provincial Education Department (15ZA0288).

\section{Appendix}

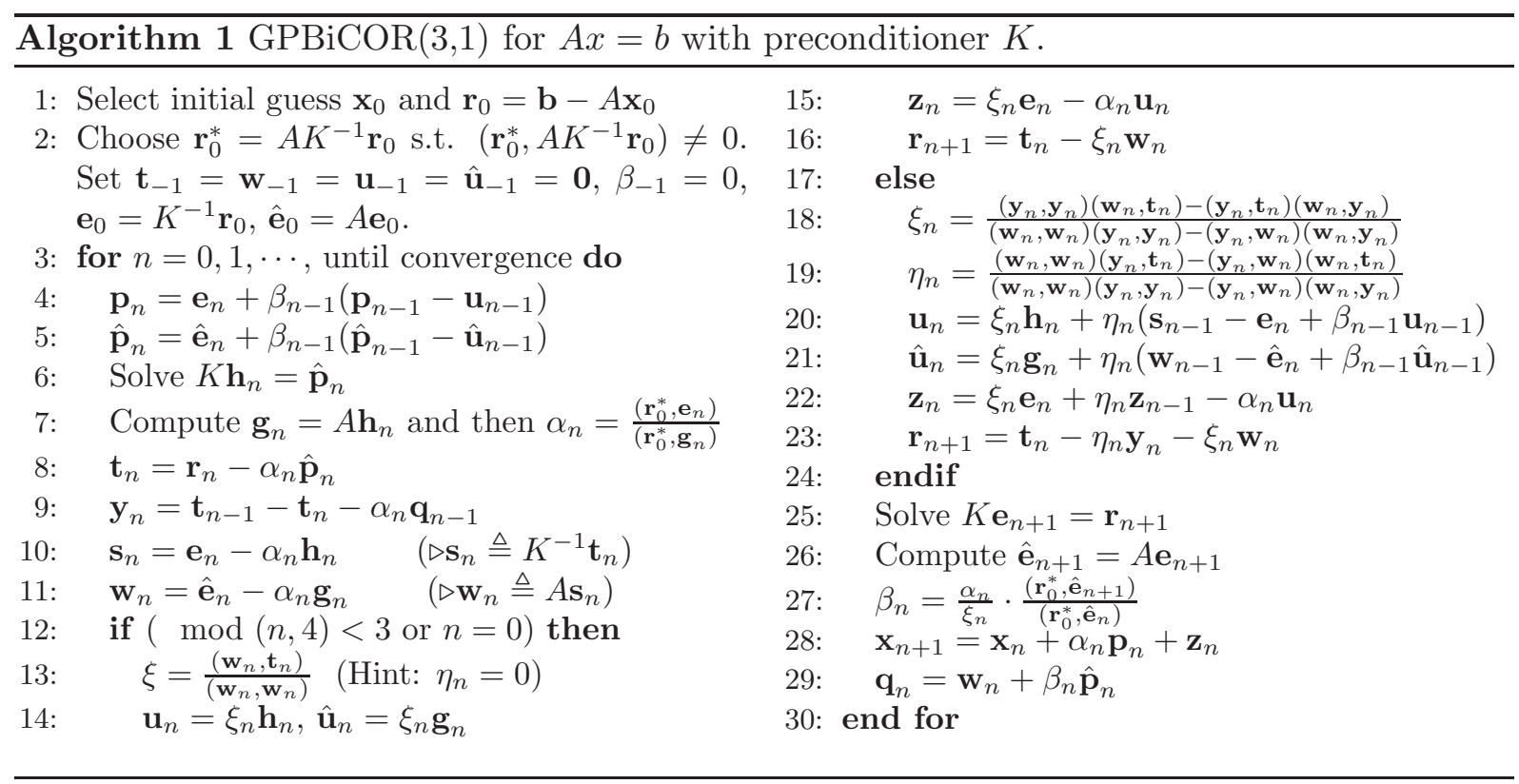

\section{References}

[1] F. Liu, P. Zhuang, V. Anh, I. Turner, K. Burrage, Stability and convergence of the difference methods for the space-time fractional advection-diffusion equation, Appl. Math. Comput., 191 (2007), pp. 1220.

[2] Z. Zhao, X.-Q. Jin, M.-M. Lin, Preconditioned iterative methods for space-time fractional advectiondiffusion equations, J. Comput. Phys., 319 (2016), pp. 266-279.

[3] I. Podlubny, Fractional Differential Equations, vol. 198 of Mathematics in Science and Engineering, Academic Press, Inc., San Diego, CA, 1999.

[4] S. G. Samko, A. A. Kilbas, O. I. Marichev, Fractional Integrals and Derivatives: Theory and Applications, Gordon and Breach Science Publishers, Yverdonn, 1993.

[5] D. A. Benson, R. Schumer, M. M. Meerschaert, S. W. Wheatcraft, Fractional dispersion, Lévy motion, and the MADE tracer tests, Transport in Porous Media, 42 (2001), pp. 211-40.

[6] D. A. Benson, S. W. Wheatcraft, M. M. Meerschaert, The fractional-order governing equation of Lévy motion, Water Resour. Res., 36 (2000), pp. 1413-23. 
[7] R. Metzler, J. Klafter, The random walks guide to anomalous diffusion: a fractional dynamics approach, Phys. Rep., 339 (2000), pp. 1-77.

[8] Y. Povstenko, Generalized boundary conditions for the time-fractional advection diffusion equation, Entropy, 17 (2015), pp. 4028-4039.

[9] D. A. Benson, S. W. Wheatcraft, M. M. Meerschaert, Application of a fractional advection-dispersion equation, Water Resour. Res., 36 (2000), pp. 1403-1412.

[10] S. Abbasbandy, S. Kazem, M. S.Alhuthali, H. H.Alsulami, Application of the operational matrix of fractional-order Legendre functions for solving the time-fractional convection-diffusion equation, Appl. Math. Comput., 266 (2015), pp. 31-40.

[11] V. J. Ervin, N. Heuer, J. P. Roop, Numerical approximation of a time dependent, nonlinear, spacefractional diffusion equation, SIAM J. Numer. Anal., 45 (2007), pp. 572-591.

[12] N. J. Ford, A. C. Simpson, The numerical solution of fractional differential equations: speed versus accuracy, Numer. Algor., 26 (2001), pp. 333-346.

[13] F. Liu, V. Anh, I. Turner, Numerical solution of the space fractional Fokker-Planck equation, $J$. Comput. Appl. Math., 166 (2004), pp. 209-219.

[14] M. M. Meerschaert, C. Tadjeran, Finite difference approximations for fractional advection-dispersion flow equations, J. Comput. Appl. Math., 172 (2004), pp. 65-77.

[15] M. M. Meerschaert, C. Tadjeran, Finite difference approximations for two-sided spacefractional partial differential equations, Appl. Numer. Math., 56 (2006), pp. 80-90.

[16] M. M. Meerschaert, H. P. Scheffler, C. Tadjeran, Finite difference methods for two-dimensional fractional dispersion equation, J. Comput. Phys., 211 (2006), pp. 249-261.

[17] H. Wang, K. Wang, T. Sircar, A direct $\mathcal{O}(N \log 2 N)$ finite difference method for fractional diffusion equations, J. Comput. Phys., 229 (2010), pp. 8095-8104.

[18] W.-H. Luo, T.-Z. Huang, G.-C. Wu, X.-M. Gu, Quadratic spline collocation method for the time fractional subdiffusion equation, Appl. Math. Comput., 276 (2016), pp. 252C265.

[19] X.-M. Gu, T.-Z. Huang, X.-L. Zhao, H.-B. Li, L. Li, Strang-type preconditioners for solving fractional diffusion equations by boundary value methods, J. Comput. Appl. Math., 277 (2015), pp. 73-86.

[20] L. Wei, X. Zhang, Y. He, Analysis of a local discontinuous Galerkin method for time fractional advection-diffusion equations, Int. J. Numer. Method. H., 23 (2013), pp. 634 - 648.

[21] P. Zhuang, Y.-T. Gu, F. Liu, I. Turner, P.K.D.V. Yarlagadda, Time-dependent fractional advectiondiffusion equations by an implicit MLS meshless method, Int. J. Numer. Meth. Eng., 88 (2011), pp. 1346-1362.

[22] F. Liu, P. Zhuang, K. Burrage, Numerical methods and analysis for a class of fractional advectiondispersion models, Comput. Math. Appl., 64 (2012), pp. 2990-3007.

[23] E. Sousa, C. Li, A weighted finite difference method for the fractional diffusion equation based on the Riemann-Liouville derivative, Appl. Num. Math., 90 (2015), pp. 22-37.

[24] W. Qu, S.-L. Lei, S.-W. Vong, Circulant and skew-circulant splitting iteration for fractional advection-diffusion equations, Int. J. Comput. Math., 91 (2014), pp. 2232-2242.

[25] Z.-J. Fu, W. Chen, H.-T. Yang, Boundary particle method for Laplace transformed time fractional diffusion equations, J. Comput. Phys., 235 (2013), pp. 52-66.

[26] S. Zhai, X. Feng, Y. He, An unconditionally stable compact ADI method for three-dimensional time-fractional convection-diffusion equation, J. Comput. Phys., 269 (2014), pp. 138-155. 
[27] Y.-M. Wang, A compact finite difference method for solving a class of time fractional convectionsubdiffusion equations, BIT, 55 (2015), pp. 1187-1217.

[28] Y. Zhang, A finite difference method for fractional partial differential equation, Appl. Math. Comput., 215 (2009), pp. 524-529.

[29] Y. Zhang, Finite difference approximations for space-time fractional partial differential equation, $J$. Numer. Math., 17 (2009), pp. 319-326.

[30] S. Shen, F. Liu, V. Anh, Numerical approximations and solution techniques for the space-time Riesz-Caputo fractional advection-diffusion equation, Numer. Algor., 56 (2011), pp. 383-403.

[31] J. Wei, Y. Chen, B. Li, M. Yi, Numerical solution of space-time fractional convection-diffusion equations with variable coefficients using Haar wavelets, Comput. Model. Eng. Sci. (CMES), 89 (2012), pp. 481-495.

[32] H. Hejazi, T. Moroney, F. Liu, A finite volume method for solving the two-sided time-space fractional advection-dispersion equation, Cent. Eur. J. Phys., 11 (2013), pp. 1275-1283.

[33] S. Irandoust-pakchin, M. Dehghan, S. Abdi-mazraeh, M. Lakestani, Numerical solution for a class of fractional convection-diffusion equations using the flatlet oblique multiwavelets, J. Vib. Control, 20 (2014), pp. 913-924.

[34] M. Parvizi, M. R. Eslahchi, M. Dehghan, Numerical solution of fractional advection-diffusion equation with a nonlinear source term, Numer. Algor., 68 (2015), pp. 601-629.

[35] M. Cui, Compact exponential scheme for the time fractional convection-diffusion reaction equation with variable coefficients, J. Comput. Phys., 280 (2015), pp. 143-163.

[36] H. Wang, K. Wang, T. Sircar, A direct $\mathcal{O}\left(N \log ^{2} N\right)$ finite difference method for fractional diffusion equations, J. Comput. Phys., 229 (2010), pp. 8095-8104.

[37] M. Ng, Iterative Methods for Toeplitz Systems, Oxford University Press, New York, 2004.

[38] K. Wang, H. Wang, A fast characteristic finite difference method for fractional advection-diffusion equations, Adv. Water Resour., 34 (2011), pp. 810-816.

[39] X.-M. Gu, T.-Z. Huang, H.-B. Li, L. Li, W.-H. Luo, On $k$-step CSCS-based polynomial preconditioners for Toeplitz linear systems with application to fractional diffusion equations, Appl. Math. Lett., 42 (2015), pp. 53-58.

[40] Y.-Q. Bai, T.-Z. Huang, X.-M. Gu, Circulant preconditioned iterations for fractional diffusion equations based on Hermitian and skew-Hermitian splittings, Appl. Math. Lett., 48 (2015), pp. 14-22.

[41] H. A. van der Vorst, Bi-CGSTAB: A fast and smoothly converging variant of Bi-CG for the solution of nonsysmetric linear systems, SIAM J. Sci. Stat. Comput., 13 (1992), pp. 631-644.

[42] X.-M. Gu, T.-Z. Huang, B. Carpentieri, L. Li, C. Wen, A hybridized iterative algorithm of the BiCORSTAB and GPBiCOR methods for solving non-Hermitian linear systems, Comput. Math. Appl., 70 (2015), pp. 3019-3031.

[43] A. A. Alikhanov, A new difference scheme for the time fractional diffusion equation, J. Comput. Phys., 280 (2015), pp. 424-438.

[44] W.-Y. Tian, H, Zhou, W.-H. Deng, A class of second order difference approximations for solving space fractional diffusion equations, Math. Comput., 84 (2015), pp. 1703-1727.

[45] L.-B. Feng, P. Zhuang, F. Liu, I. Turner, J. Li, High-order numerical methods for the Riesz space fractional advection-dispersion equations, Comput. Math. Appl., (2016). doi:10.1016/j.camwa.2016.01.015. 
[46] A. Mohebbi, M. Abbaszadeh, M. Dehghan, A high-order and unconditionally stable scheme for the modified anomalous fractional sub-diffusion equation with a nonlinear source term, J. Comput. Phys., 240 (2013), pp. 36-48.

[47] M. Dehghan, M. Safarpoor, M. Abbaszadeh, Two high-order numerical algorithms for solving the multi-term time fractional diffusion-wave equations, J. Comput. Appl. Math., 290 (2015), pp. 174195.

[48] S.-L. Lei, H.-W. Sun, A circulant preconditioner for fractional diffusion equations, J. Comput. Phys., 242 (2013), pp. 715-725.

[49] R. Chan, X. Jin, An Introduction to Iterative Toeplitz Solvers, SIAM, Philadelphia, 2007.

[50] X.-M. Gu, T.-Z. Huang, C.-C. Ji, B. Carpentieri, A. A. Alikhanov, Fast iterative method with a second-order implicit difference scheme for time-space fractional convection-diffusion equation, $J$. Sci. Comput. 72 (2017) 957-985. 